\title{
The INDIA Mutations and B.1.617 Variant: Is there a global "strategy" for mutations and evolution of variants of the SARS-CoV2 genome?
}

\begin{abstract}
Jean-Claude Perez, PhD Maths § Computer Science Bordeaux University, RETIRED interdisciplinary researcher (IBM Emeritus, IBM European Research Center on Artificial Intelligence Montpellier), Bordeaux metropole, France, jeanclaudeperez2@gmail.com
\end{abstract}

\begin{abstract}
.
In this paper, we run for all INDIA mutations and variants a biomathematical numerical method for analysing mRNA nucleotides sequences based on UA/CG Fibonacci numbers proportions (Perez, 2021). In this study, we limit ourselves to the analysis of whole genomes, all coming from the mutations and variants of SARS-CoV2 sequenced in India in 2020 and 2021. We then demonstrate - both on actual genomes of patients and on variants combining the most frequent mutations to the SARS-CoV2 Wuhan genomes and then to the B.1.617 variant - that the numerical Fibonacci AU / CG metastructures increase considerably in all cases analyzed in ratios of up to 8 times. We can affirm that this property contributes to a greater stability and lifespan of messenger RNAs, therefore, possibly also to a greater INFECTUOSITY of these variant genomes.
\end{abstract}

\section{I - INTRODUCTION.}

After various papers related SARS-CoV2 origins and evolution (Perez, 2020) and (Perez§Montagnier, 2020), in (Perez, 2021), we presented a biomathematical method based on mRNA genomes and spikes UA/CG Fibonacci nucleotides proportions. Particularly we demonstrated a real corelation between variants evolution (UK, South Africa, California, Brazil) and the amount of long range Fibonacci metastructures.

In order to test this hypothesis, we are interested in the 2 countries in which the effect of variants seems uncontrollable: Brazil and India.

We chose India because the sequencing of genomes is more systematic and reliable there than in Brazil.

For this we proceed in 2 steps:

- Analyzing the first variants of 2020 . For this we rely on this publication:

(Muttineri et al, 2021),

https://www.google.com/url?sa=t\&source=web\&rct=j\&url=https://journals.plos.org/plospathogens/article/file \%3Fid\%3D10.1371/journal.pone.0246173\%26type

\%3Dprintable\&ved=2ahUKEwj3zdnZnorwAhUQKBoKHUxnD EQFjABegQICBAC\&usg=AOvVaw1A79ux6Ub etoPoRx_jT-Mk

2/ Then we study the most recent changes of 2021 . For that we rely on this sydtematic approach:

(Srivastava Surabhi et al, 2021), https://www.ncbi.nlm.nih.gov/pmc/articles/PMC7895735/ And more particularly on this Indian GEAR19 database:

https://data.ccmb.res.in/gear19/variants

\section{II- METHODS and DATA SOURCES.}

\section{1 - Computing FIBONACCI metastructures:}

Consider the sequence of Fibonacci numbers 
0112358132134558914423337761098715972584418167651094617711 28657463687502512139319641831781151422983204013462692178309 $35245785702887 \ldots$

Example of the SPIKE from Wuhan reference genome, this mRNA SPIKE is 3822 bases UCAG in length.

Recall Wuhan reference https://www.ncbi.nlm.nih.gov/nuccore/NC 045512

Severe acute respiratory syndrome coronavirus 2 isolate Wuhan-Hu-1, complete genome NCBI Reference Sequence: NC_045512.2

the longest Fibonacci structures would therefore measure 2584 bases.

When looking for such structures, the first one found is in 1200 location:

therefore, the bases located between 1201 and $3784(1200+2584)$ :

These 2584 bases are broken down respectively into:

1597 bases UA

et 987 bases $\mathrm{CG}$

Here are the first 20 basics that the reader can easily check:

SPIKREF[1200+1/420]

G U A A UU A G A G G U G A U G A A G U

$\begin{array}{llllllllllllllllllllll}0 & 1 & 1 & 1 & 1 & 1 & 1 & 0 & 1 & 0 & 0 & 1 & 0 & 1 & 1 & 0 & 1 & 1 & 0 & 1 \ldots / \ldots\end{array}$

U AAUUA A U A U A A U 1597 bases UA

G UAA UU A G A G G U G A U G A A G U

$\begin{array}{lllllllllllllllllllll}1 & 0 & 0 & 0 & 0 & 0 & 0 & 1 & 0 & 1 & 1 & 0 & 1 & 0 & 0 & 1 & 0 & 0 & 1 & 0 \ldots\end{array}$

$\begin{array}{lllllll}G & G & G G & G & G & G & 987 \text { bases }\end{array}$

The SPIKE analyzes of this Wuhan-Hu-1 reference genome reports 63 metastructures of this type if we close the sequence on itself (as in mtDNA or bacteria) and 7 metastructures and if we consider the mRNA sequence in its linear form, as will be the case throughout this study.

\section{2 - Analyzes of reference variants :}

\subsection{1/ Analyzing the first variants of 2020 :}

- Analyzing the first variants of 2020 . For this we rely on this publication:

(Muttineri et al, 2021),

https://www.google.com/url?sa=t\&source=web\&rct=j\&url=https://journals.plos.org/plospathogens/article/file \%3Fid\%3D10.1371/journal.pone.0246173\%26type

\%3Dprintable\&ved=2ahUKEwj3zdnZnorwAhUQKBoKHUxnD_EQFjABegQICBAC\&usg=AOvVaw1A79ux6Ub etoPoRx jT-Mk

The full-genome viral sequences were deposited in the dataset of GISAID (EPI_ISL_431101, EPI_ISL_431102, EPI_ISL_431103, EPI_ISL_431117, EPI_ISL_438139, EPI_ISL_437626, EPI_ISL_438138) and NCBI GenBank (MT415320, MT415321, MT415322, MT415323, MT47778̄5, MT457402, MT457403).

Now we analyse :

GenBank (MT415320, MT415321, MT415322, MT415323, MT477885, MT457402, MT457403)

Main data source for mutations : https://covariants.org/ 


\subsection{2/ Analyzing 28 INDIAN mutations applied to SARS-CoV2 Wuhan reference genome :}

Then we study the most recent changes of 2021. For that we rely on this systematic approach:

(Srivastava Surabhi et al, 2021), https://www.ncbi.nlm.nih.gov/pmc/articles/PMC7895735/

And more particularly on this Indian GEAR19 database:

https://data.ccmb.res.in/gear19/variants

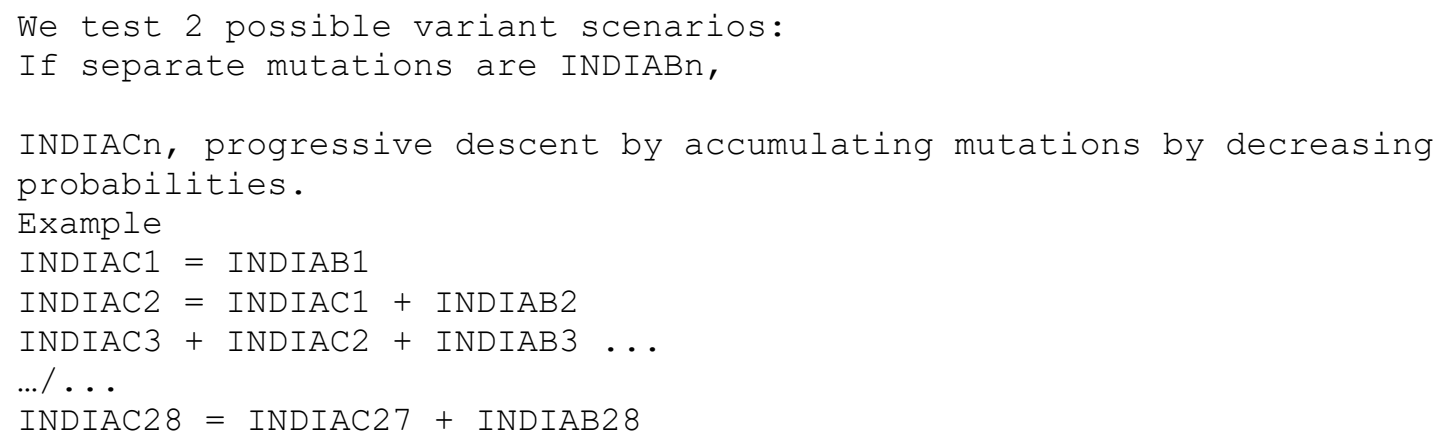

link Table 5\%

https://mail.google.com/mail/u/0/\#inbox/KtbxLzGLmpFSTVtcKRqRlmnxKrplVzgNnq? projector $=1 \&$ messagePartId $=0.1$

\subsection{3/ Analyzing 28 INDIAN mutations applied to B.1.617 India variant genome :}

We run the same 28 genomes simulations starting from the India variant B.1.617.

\subsection{4/ simulations of possible future mutations of the variant B.1.617.}

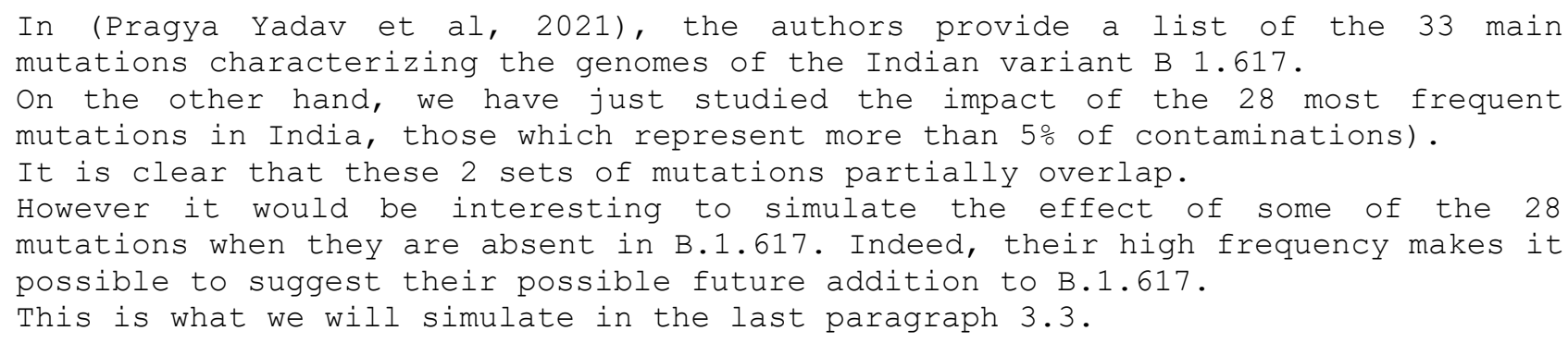

\section{III - RESULTS and DISCUSSION.}

\section{1 - Analyzing the first variants of 2020 :}


Now we analyse from (Muttineri et al, 2021) :

GenBank (MT415320, MT415321, MT415322, MT415323, MT477885, MT457402, MT457403)

INDIAA1 MT415320

https://www.ncbi.nlm.nih.gov/nuccore/MT415320

INDIAA2 MT415321

https://www.ncbi.nlm.nih.gov/nuccore/MT415321

INDIAA3 MT415322

https://www.ncbi.nlm.nih.gov/nuccore/MT415322

INDIAA4 MT415323

https://www.ncbi.nlm.nih.gov/nuccore/MT415323

INDIAA5 MT457402

https://www.ncbi.nlm.nih.gov/nuccore/MT457402

INDIAA6 MT457403

https://www.ncbi.nlm.nih.gov/nuccore/MT457403

INDIA7 MT477885

https://www.ncbi.nlm.nih.gov/nuccore/MT477885

Table 1 - Mutations Table from paper https://journals.plos.org/plosone/article/figure? $\underline{\mathrm{id}=10.1371 / \text { journal.pone. } 0246173 . t 003}$

\begin{tabular}{|c|c|c|c|c|c|c|c|c|c|c|c|c|c|c|c|c|c|c|c|c|c|c|c|c|c|c|c|c|}
\hline & \multicolumn{12}{|c|}{ ORF1 ab } & \multicolumn{8}{|c|}{ Spike } & \multicolumn{2}{|l|}{ ORF3a } & \multicolumn{6}{|c|}{ Nucleocapsid } \\
\hline & $\mathrm{N}$ & AA & $\mathrm{N}$ & AA & $\mathrm{N}$ & AA & $\mathrm{N}$ & AA & $\mathrm{N}$ & AA & $\mathrm{N}$ & AA & $\mathrm{N}$ & AA & $\mathrm{N}$ & AA & $\mathrm{N}$ & AA & $\mathrm{N}$ & AA & $\mathrm{N}$ & AA & $\mathrm{N}$ & AA & $\mathrm{N}$ & AA & $\mathrm{N}$ & AA \\
\hline & 3037 & 925 & 6312 & 2,016 & 11,083 & 3,606 & 13,730 & 4,489 & 14,408 & 4,715 & 15,324 & 5,020 & 18,877 & 6205 & 21644 & 28 & 23403 & 614 & 23929 & 789 & 24130 & 856 & 25563 & 57 & 28311 & 13 & 29303 & 344 \\
\hline Wuhan $\mathrm{Hu}-1$ & C & F & C & $\mathrm{T}$ & G & L & C & A & C & $\mathbf{P}$ & $\mathrm{C}$ & $\mathbf{N}$ & C & L & $\mathrm{T}$ & $\mathrm{Y}$ & A & D & C & $\mathrm{Y}$ & C & $\mathrm{N}$ & G & Q & C & $\mathbf{P}$ & C & $\mathbf{P}$ \\
\hline OUMRK 100/2020 & C & F & C & $\mathrm{T}$ & G & L & C & A & C & $\mathbf{P}$ & $\mathrm{T}$ & $\mathrm{N}$ & C & L & C & H & A & D & C & $\mathrm{Y}$ & $\mathrm{T}$ & N & G & Q & C & $\mathbf{P}$ & $T$ & $\mathrm{~s}$ \\
\hline GMCKN318/2020 & T & F & C & $\mathrm{T}$ & G & L & C & A & $T$ & L & C & $\mathbf{N}$ & C & L & $\mathrm{T}$ & $\mathrm{Y}$ & G & G & C & $\mathbf{Y}$ & C & $\mathbf{N}$ & G & Q & C & $\mathbf{P}$ & C & $P$ \\
\hline GMCKN443/2020 & C & F & C & K & $\mathrm{T}$ & F & $\mathrm{T}$ & G & C & $\mathbf{P}$ & C & $\mathbf{N}$ & C & L & $\mathrm{T}$ & $\mathbf{Y}$ & A & D & $\mathrm{T}$ & $\mathbf{Y}$ & C & $\mathbf{N}$ & G & Q & $\mathrm{T}$ & $\mathbf{L}$ & C & $\mathbf{P}$ \\
\hline GMCTC469/2020 & $\mathrm{T}$ & F & C & $\mathrm{T}$ & G & L & C & A & $\mathrm{T}$ & L & C & $\mathrm{N}$ & C & L & $\mathrm{T}$ & $\mathrm{Y}$ & G & G & C & $\mathrm{Y}$ & C & $\mathrm{N}$ & G & Q & C & $\mathbf{P}$ & C & P \\
\hline OUMRK 1090/2020 & $\mathrm{c}$ & F & A & $\mathrm{N}$ & $\mathrm{T}$ & F & $\mathrm{T}$ & $\mathrm{v}$ & C & P & C & $\mathbf{N}$ & C & $\mathbf{L}$ & $\mathrm{T}$ & $\mathrm{Y}$ & A & D & $\mathrm{T}$ & $\mathbf{Y}$ & C & $\mathrm{N}$ & G & Q & $\mathrm{T}$ & L & C & $\mathbf{P}$ \\
\hline GMCKP1125/2020 & $\mathrm{T}$ & F & C & $\mathrm{T}$ & G & L & C & A & $\mathrm{T}$ & L & C & N & $\mathrm{T}$ & L & $\mathrm{T}$ & $\mathrm{Y}$ & G & G & C & $\mathrm{Y}$ & C & N & T & H & C & $\mathbf{P}$ & C & $\mathbf{P}$ \\
\hline GMC-RR1191/2020 & C & F & A & K & $T$ & F & $\mathrm{T}$ & $\mathrm{v}$ & C & P & C & $\mathbf{N}$ & C & L & $\mathrm{T}$ & $\mathrm{Y}$ & A & D & $\mathrm{T}$ & $\mathrm{Y}$ & C & $\mathbf{N}$ & G & Q & T & L & C & P \\
\hline
\end{tabular}

https://doi.org/10.1371/journal.pone.0246173.t003

https://journals.plos.org/plosone/article/figure?id=10.1371/journal.pone.0246173.t003

Table 2 - Summary Table related 7 SARS-CoV2 real patients from India sequenced in 2020.

\begin{tabular}{|l|l|l|l|l|}
\hline Reference & $\begin{array}{l}\text { Alias number line } \\
\text { in Table1 }\end{array}$ & $\begin{array}{l}\text { GENBANK } \\
\text { Identification }\end{array}$ & Date & $\begin{array}{l}\text { Number of 17711 } \\
\text { UA/CG } \\
\text { metastructures }\end{array}$ \\
\hline $\begin{array}{l}\text { SARS-CoV2 } \\
\text { Wuhan }\end{array}$ & NC_045512.2 & 18-JUL-2020 & 8 \\
\hline INDIAA1 & $\begin{array}{l}\text { INDIAA1 } \\
\text { MT415320 line1 }\end{array}$ & MT415320.1 & 30 -APR-2020 & 23 \\
\hline INDIAA2 & $\begin{array}{l}\text { INDIAA2 } \\
\text { MT415321 line2 }\end{array}$ & MT415321.1 & 30 -APR-2020 & 8 \\
\hline INDIAA3 & $\begin{array}{l}\text { INDIAA3 } \\
\text { MT415322 line3 }\end{array}$ & MT415322.1 & $30-$ APR-2020 & 33 \\
\hline INDIAA4 & $\begin{array}{l}\text { INDIAA4 } \\
\text { MT415323 line4 }\end{array}$ & MT415323.1 & $30-$ APR-2020 & 45 \\
\hline INDIAA5 & $\begin{array}{l}\text { INDIAA5 } \\
\text { MT457402 line6 }\end{array}$ & MT457402.1 & 12 -MAY-2020 & 45 \\
\hline
\end{tabular}




\begin{tabular}{|l|l|l|l|l|}
\hline INDIAA6 & $\begin{array}{l}\text { INDIAA5 } \\
\text { MT457402 line7 }\end{array}$ & MT457403.1 & 12-MAY-2020 & 34 \\
\hline INDIAA7 & $\begin{array}{l}\text { INDIA7 } \\
\text { MT477885 line5 }\end{array}$ & MT477885.1 & 18-MAY-2020 & 37 \\
\hline
\end{tabular}

Genomes lengths :

VSARSCOV2REF 29903

VINDIAA1 29900

VINDIAA2 29903

VINDIAA3 29888

VINDIAA4 29890

VINDIAA5 29890

VINDIAA6 29890

VINDIAA7 29899

6 of the 7 cases have deletions.

Only INDIAA2 has the same length as SARS-CoV2 Wuhan reference.

VSARSCOV2REF 29903

VINDIAA2 29903

Only 4 difference bases: it is precisely the only one that has not increased the number of metastructures.

Number of diferent bases : +/VSARSCOV2REF $\neg$ VINDIAA2 $=4$

Locations : (VSARSCOV2REF $\neg$ VINDIAA2)/1/4 $1 / 2$ VINDIAA2

24130371440823403

Nucleotides values in SARS-CoV2 ref : $\quad$ (VSARSCOV2REF $\neg$ VINDIAA2)/VSARSCOV2REF CCCA

Nucleotides values in VINDIAA2 : $\quad$ (VSARSCOV2REF $\neg$ VINDIAA2)/VINDIAA2

TTTG

i.e. 3 out of 4 CG mutations $==>$ UA

From the results below I deduce that the deletions of 5 cases out of 6 studied cases

contributed to considerably increase the UA/ CG metastructures of 17711 bases. 


\section{Whole GENOME SARS-CoV2 WUHAN \\ FIBONACCI UCAG Metastructures}

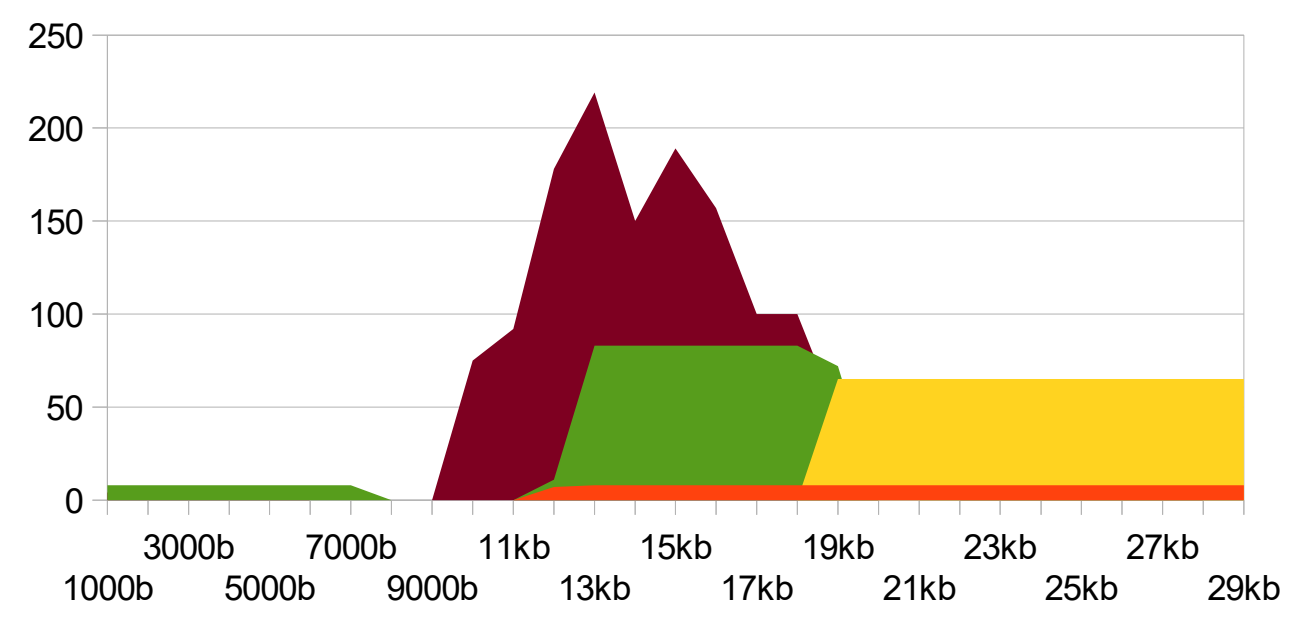

- 28657b

- 17711b

$10946 \mathrm{~b}$

- 6765b

- 4181b

Figure 1 - Recall SARS-CoV2 Wuhan genome metastructures.

\section{INDIAA1}

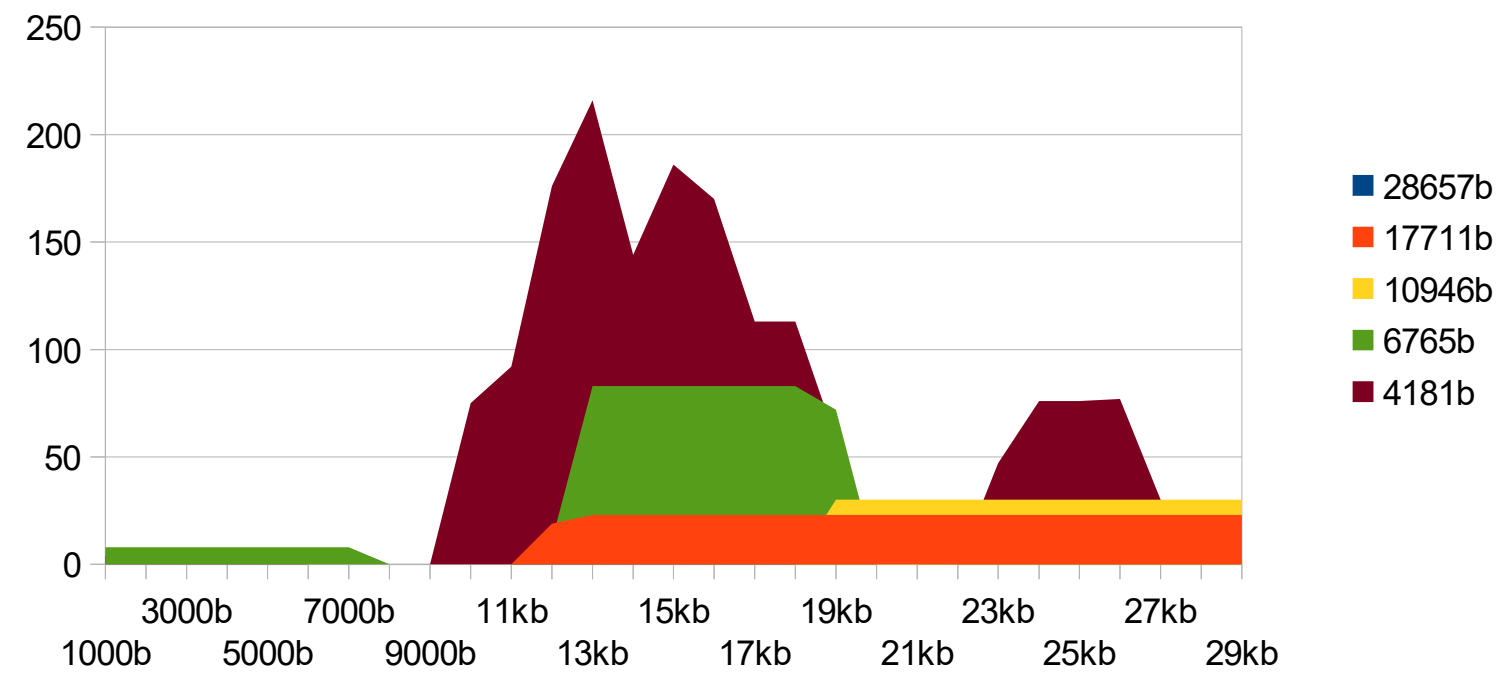

Figure 2 - INDIAA1 genome metastructures. 


\section{INDIAA2}

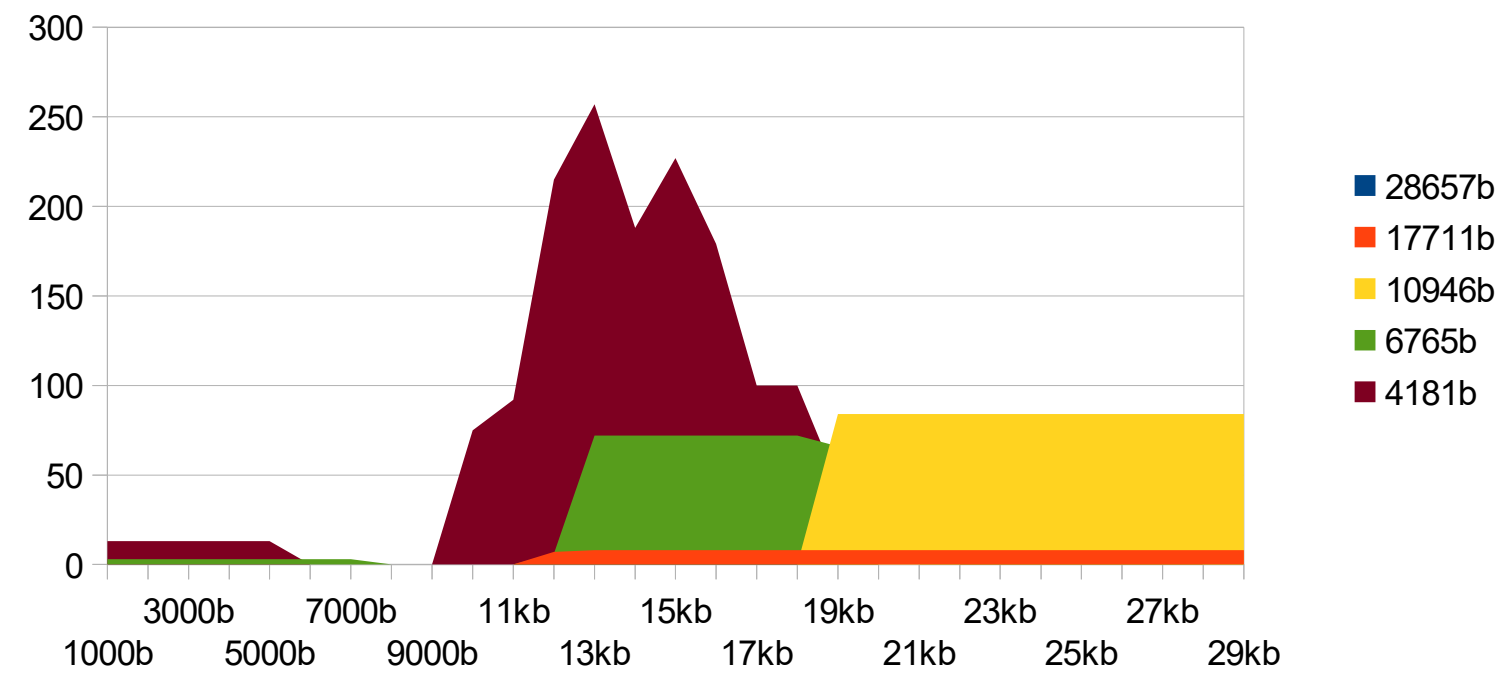

Figure 3 - INDIAA2 genome metastructures.

\section{INDIAA3}

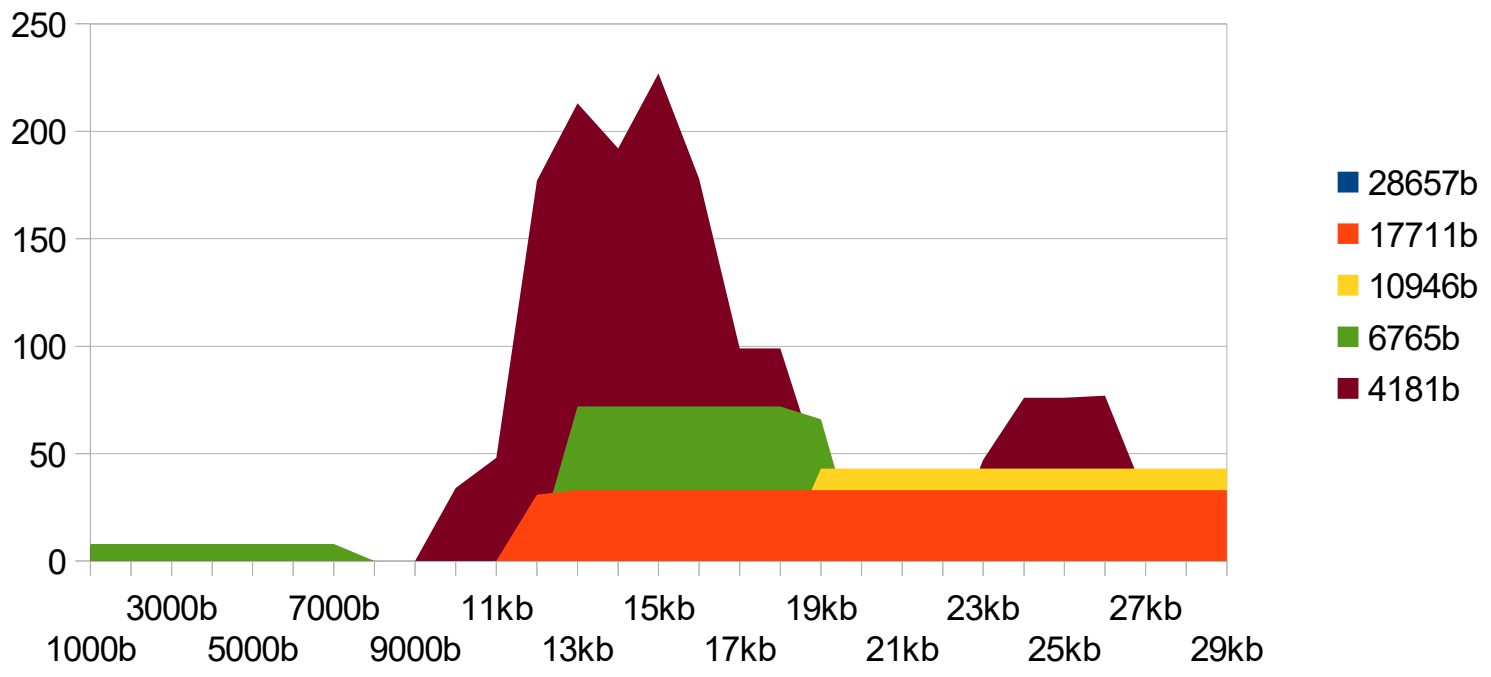

Figure 4 - INDIAA3 genome metastructures. 


\section{INDIAA4}

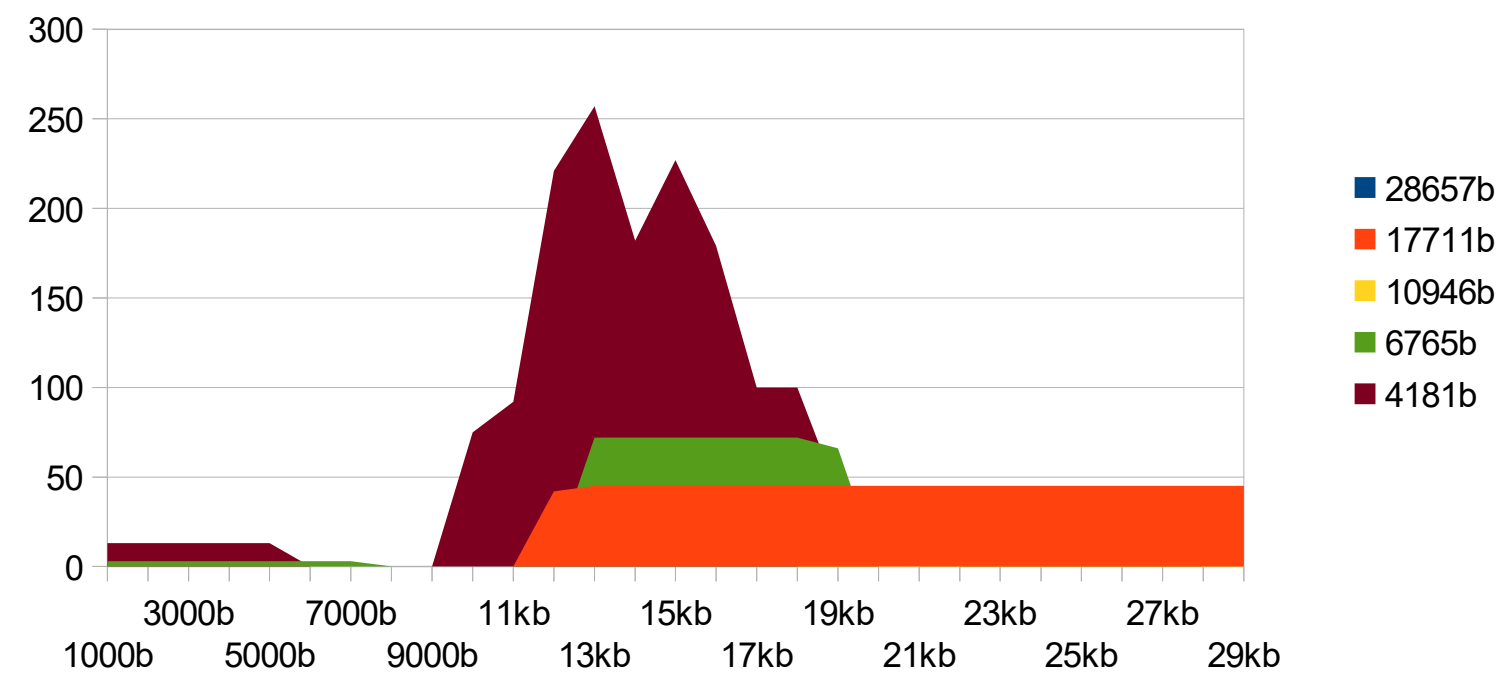

Figure 5 - INDIAA4 genome metastructures.

INDIAA5

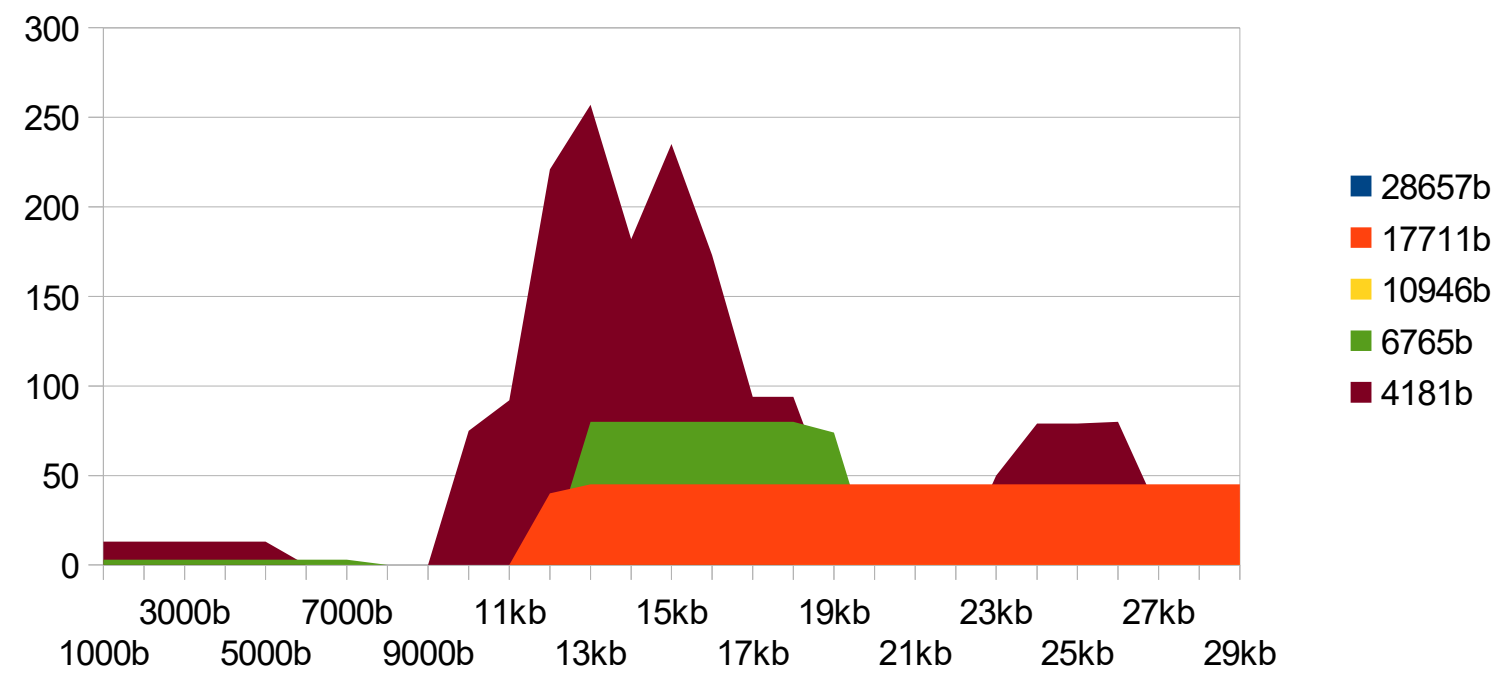

Figure 6 - INDIAA5 genome metastructures. 


\section{INDIAA6}

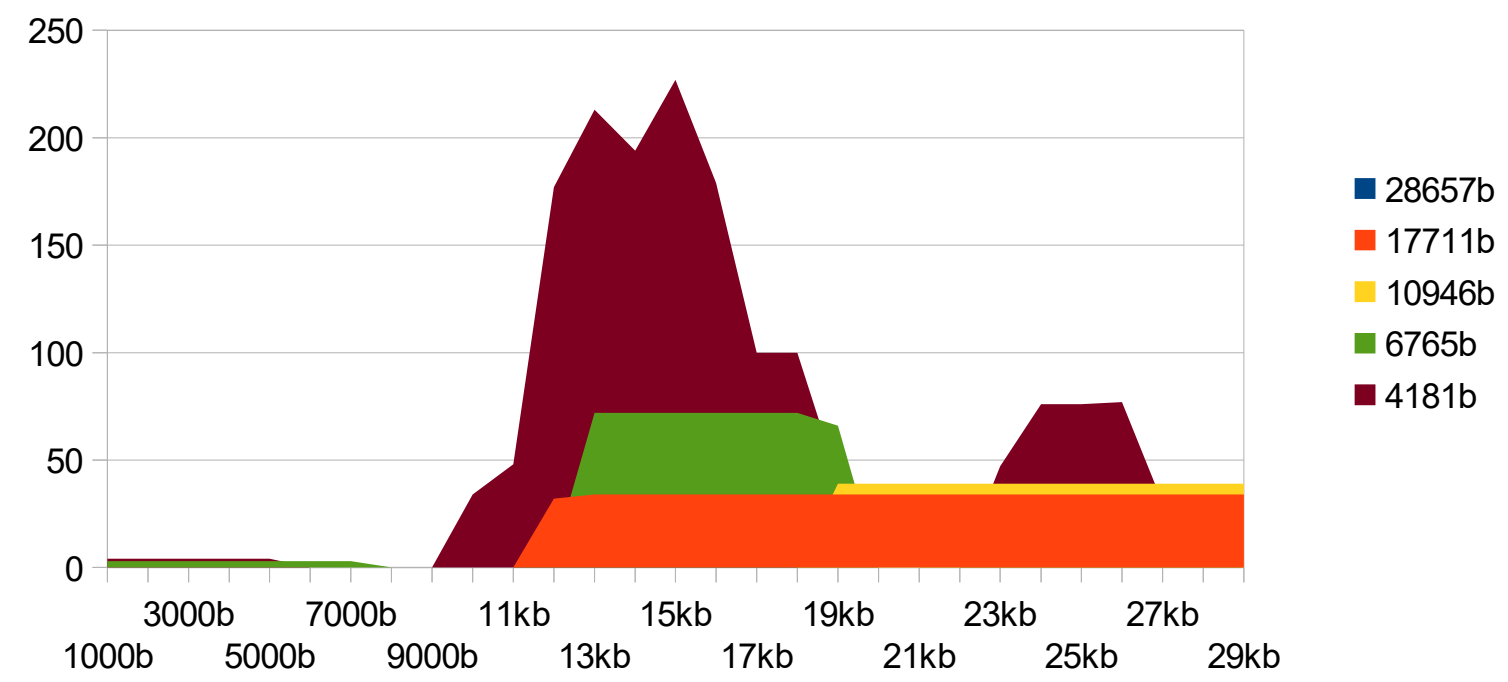

Figure 7 - INDIAA6 genome metastructures.

\section{INDIAA7}

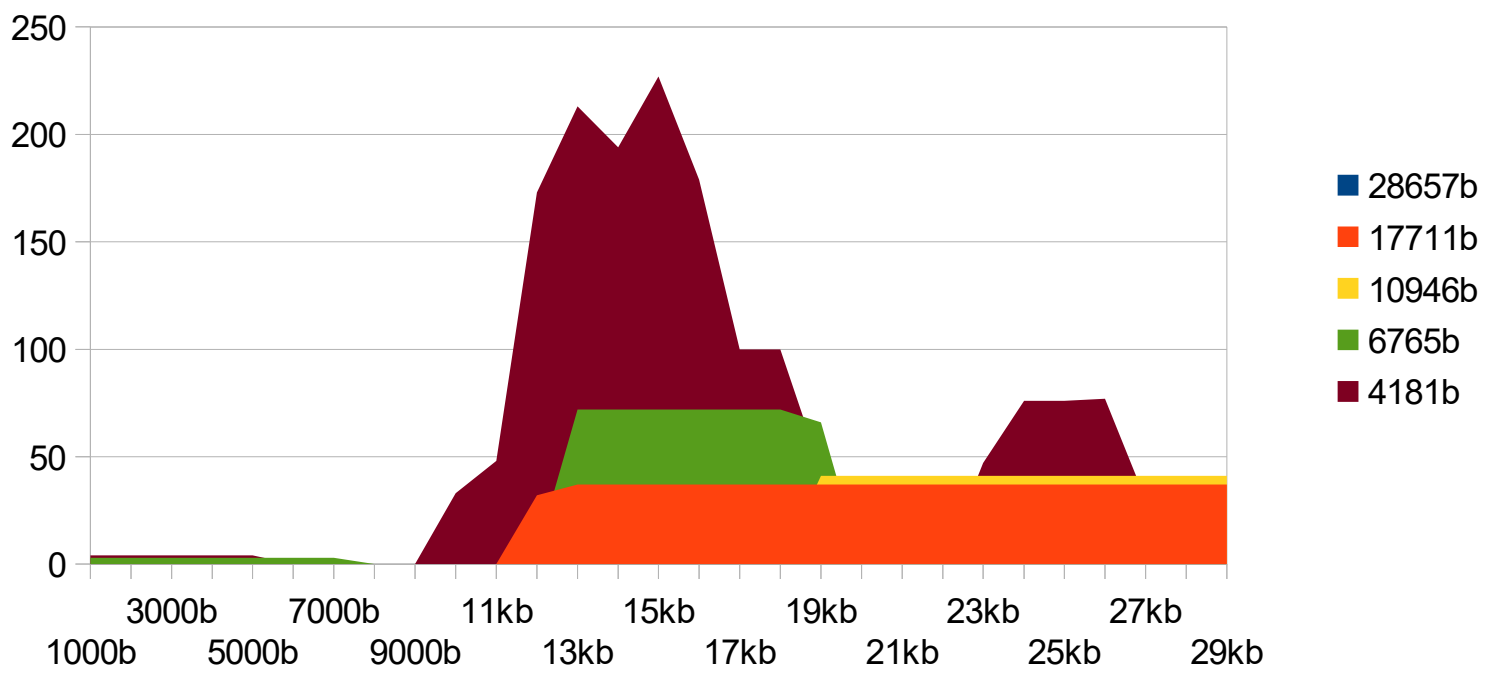

Figure 8 - INDIAA7 genome metastructures.

\section{2 - INDIAN VARIANTS SIMULATIONS with mutations on SARS-CoV2 Wuhan :}

We work now from these published data :

We test 2 possible variant scenarios: (Srivastava Surabhi et al, 2021), and more particularly on this Indian GEAR19 database: https://data.ccmb.res.in/gear19/variants

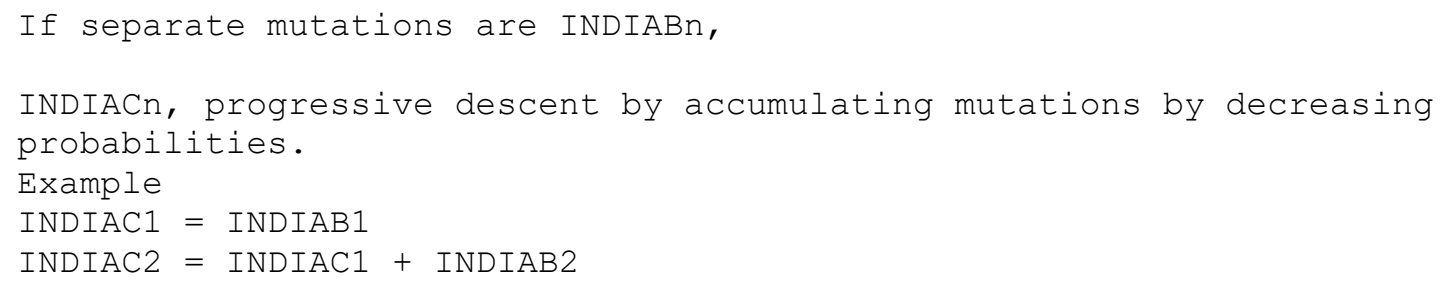


INDIAC3 + INDIAC2 + INDIAB3 $\ldots$

.../...

INDIAC28 $=$ INDIAC27 + INDIAB28

Then we study the most recent changes of 2021. For that we rely on this systematic approach:

https://www.ncbi.nlm.nih.gov/pmc/articles/PMC7895735/

And more particularly on this Indian GEAR19 database:

\section{https://data.ccmb.res.in/gear19/variants}

link Table 5\% :

https://mail.google.com/mail/u/0/\#inbox/KtbxLzGLmpFSTVtcKRqRlmnxKrplVzgNnq? projector $=1 \&$ messagePartId $=0.1$

On the table of 28 Indian mutations $>5 \%$ of cases, The progressive study of the 29 genomes by integrating mutations step by step according to their frequency should give very interesting Fibonacci on the scale of the whole genome.

INDIAC

INDIACn, progressive descent by accumulating mutations by decreasing probabilities.

Example INDIAC2 = INDIAC1 + INDIAB2

APL Language session mutations...
$=====$ INDIAC $====$
VINDIAC1,,VSARSCOV2REF
VINDIAC1[23403],,'G'
VINDIAC2,,VINDIAC1
VINDIAC2[3037],,'T'
VINDIAC3,,VINDIAC2
VINDIAC3[241],'T'
VINDIAC4,,VINDIAC3
VINDIAC4[14408],,'T'
VINDIAC5,,VINDIAC4
VINDIAC5[28881],,'A'
VINDIAC6,,VINDIAC5
VINDIAC6[28883],,'C'
VINDIAC7,,VINDIAC6
VINDIAC7[28882],,'A'
VINDIAC8,,VINDIAC7
VINDIAC8[25563],'T'
VINDIAC9,,VINDIAC8
VINDIAC9[18877],,'T'
VINDIAC10,,VINDIAC9
VINDIAC10[26735],,'T'
VINDIAC11,,VINDIAC10
VINDIAC11[28854],,'T'
VINDIAC12,,VINDIAC11
VINDIAC12[22444],,'T'
VINDIAC13,,VINDIAC12
VINDIAC13[313],,'T'
VINDIAC14,,VINDIAC13
VINDIAC14[5700],,'A'
VINDIAC15,,VINDIAC14
VINDIAC15[11083],,'T'
VINDIAC16,,VINDIAC15
VINDIAC16[13730],,'T' 
VINDIAC17,,VINDIAC16

VINDIAC17[28311],'T'

VINDIAC18,,VINDIAC17

VINDIAC18[23929],,'T'

VINDIAC19,,VINDIAC18

VINDIAC19[6312],'A'

VINDIAC20,,VINDIAC19

VINDIAC20[8917],,'T'

VINDIAC21,,VINDIAC20

VINDIAC21[1947],,'C'

VINDIAC22,,VINDIAC21

VINDIAC22[9389],,'A'

VINDIAC23,,VINDIAC22

VINDIAC23[6573],,'T'

VINDIAC24,,VINDIAC23

VINDIAC24[4354],,'A'

VINDIAC25,,VINDIAC24

VINDIAC25[25528],,'T'

VINDIAC26,,VINDIAC25

VINDIAC26[15324],,'T'

VINDIAC27,,VINDIAC26

VINDIAC27[3267],,'T'

VINDIAC28,,VINDIAC27

VINDIAC28[3634],'T'

Table 3 - Summary on the 28 most frequent India country mutations applied to SARS-CoV2 Wuhan genome.

\begin{tabular}{|l|l|l|l|l|l|l|l|}
\hline position & $\begin{array}{l}\text { Genome } \\
\text { location }\end{array}$ & ref & alt & gene & $\begin{array}{l}\text { Amino } \\
\text { Acids } \\
\text { mutations }\end{array}$ & Percent & $\begin{array}{l}\text { Number of } \\
17711 \\
\text { UA/CG } \\
\text { metastructur } \\
\text { es }\end{array}$ \\
\hline $\begin{array}{l}\text { SARS-CoV2 } \\
\text { Wuhan }\end{array}$ & & & & & & 8 \\
\hline INDIAC1 & 23403 & $\mathrm{~A}$ & $\mathrm{G}$ & "S:614" & "D614G" & 85 & 8 \\
\hline INDIAC2 & 3037 & $\mathrm{C}$ & $\mathrm{T}$ & "ORF1a:924" & "F924F" & 84 & 8 \\
\hline INDIAC3 & 241 & $\mathrm{C}$ & $\mathrm{T}$ & "5'UTR" & "NA" & 84 & 8 \\
\hline INDIAC4 & 14408 & $\mathrm{C}$ & $\mathrm{T}$ & "ORF1b:314" & "P314L" & 84 & 8 \\
\hline INDIAC5 & 28881 & $\mathrm{G}$ & $\mathrm{A}$ & "N:203" & "R203K" & 42 & 31 \\
\hline INDIAC6 & 28883 & $\mathrm{G}$ & $\mathrm{C}$ & "N:204" & "G204R" & 41 & 31 \\
\hline INDIAC7 & 28882 & $\mathrm{G}$ & $\mathrm{A}$ & "N:203" & "R203K" & 40 & 46 \\
\hline INDIAC8 & 25563 & $\mathrm{G}$ & $\mathrm{T}$ & "ORF3a:57" & "Q57H" & 25 & 35 \\
\hline INDIAC9 & 18877 & $\mathrm{C}$ & $\mathrm{T}$ & "ORF1b:1804" & "L1804L" & 25 & 25 \\
\hline INDIAC10 & 26735 & $\mathrm{C}$ & $\mathrm{T}$ & "M:71" & "Y71Y" & 25 & 10 \\
\hline INDIAC11 & 28854 & $\mathrm{C}$ & $\mathrm{T}$ & "N:194" & "S194L" & 23 & 10 \\
\hline INDIAC12 & 22444 & $\mathrm{C}$ & $\mathrm{T}$ & "S:294" & "D294D" & 22 & 8 \\
\hline INDIAC13 & 313 & $\mathrm{C}$ & $\mathrm{T}$ & "ORF1a:16" & "L16L" & 21 & 8 \\
\hline INDIAC14 & 5700 & $\mathrm{C}$ & $\mathrm{A}$ & "ORF1a:1812" & "A1812D" & 20 & 8 \\
\hline
\end{tabular}




\begin{tabular}{|l|l|l|l|l|l|l|l|}
\hline INDIAC15 & 11083 & $\mathrm{G}$ & $\mathrm{T}$ & "ORF1a:3606" & "L3606F" & 14 & 8 \\
\hline INDIAC16 & 13730 & $\mathrm{C}$ & $\mathrm{T}$ & "ORF1b:88" & "A88V" & 11 & 29 \\
\hline INDIAC17 & 28311 & $\mathrm{C}$ & $\mathrm{T}$ & "N:13" & "P13L" & 10 & 29 \\
\hline INDIAC18 & 23929 & $\mathrm{C}$ & $\mathrm{T}$ & "S:789" & "Y789Y" & 10 & 41 \\
\hline INDIAC19 & 6312 & $\mathrm{C}$ & $\mathrm{A}$ & "ORF1a:2016" & "T2016K" & 10 & 41 \\
\hline INDIAC20 & 8917 & $\mathrm{C}$ & $\mathrm{T}$ & "ORF1a:2884" & "F2884F" & 10 & 41 \\
\hline INDIAC21 & 1947 & $\mathrm{~T}$ & $\mathrm{C}$ & "ORF1a:561" & "V561A" & 7 & 41 \\
\hline INDIAC22 & 9389 & $\mathrm{G}$ & $\mathrm{A}$ & "ORF1a:3042" & "D3042N" & 6 & 41 \\
\hline INDIAC23 & 6573 & $\mathrm{C}$ & $\mathrm{T}$ & "ORF1a:2103" & "S2103F" & 6 & 41 \\
\hline INDIAC24 & 4354 & $\mathrm{G}$ & $\mathrm{A}$ & "ORF1a:1363" & "E1363E" & 6 & 41 \\
\hline INDIAC25 & 25528 & $\mathrm{C}$ & $\mathrm{T}$ & "ORF3a:46" & "L46F" & 6 & 48 \\
\hline INDIAC26 & 15324 & $\mathrm{C}$ & $\mathrm{T}$ & "ORF1b:619" & "N619N" & 6 & 36 \\
\hline INDIAC27 & 3267 & $\mathrm{C}$ & $\mathrm{T}$ & "ORF1a:1001" & "T1001I" & 6 & 36 \\
\hline INDIAC28 & 3634 & $\mathrm{C}$ & $\mathrm{T}$ & "ORF1a:1123" & "N1123N" & 6 & 36 \\
\hline Average & & & & & & $\mathbf{2 6 . 2 5 \%}$ & $\mathbf{2 6 . 8 9}$ \\
\hline
\end{tabular}

Table 4 - Recall summary main results from Table3. 


\begin{tabular}{l|r|r|} 
Genome & percent & Number of 1 \\
\hline SARS-CoV2 Wuhan & 85 & 8 \\
\hline INDIAC1 & 84 & 8 \\
\hline INDIAC2 & 84 & 8 \\
\hline INDIAC3 & 84 & 8 \\
\hline INDIAC4 & 42 & 31 \\
\hline INDIAC5 & 41 & 31 \\
\hline INDIAC6 & 40 & 46 \\
\hline INDIAC7 & 25 & 35 \\
\hline INDIAC8 & 25 & 25 \\
\hline INDIAC9 & 25 & 10 \\
\hline INDIAC10 & 23 & 10 \\
\hline INDIAC11 & 22 & 8 \\
\hline INDIAC12 & 21 & 8 \\
\hline INDIAC13 & 20 & 8 \\
\hline INDIAC14 & 14 & 8 \\
\hline INDIAC15 & 11 & 29 \\
\hline INDIAC16 & 10 & 29 \\
\hline INDIAC17 & 10 & 41 \\
\hline INDIAC18 & 10 & 41 \\
\hline INDIAC19 & 10 & 41 \\
\hline INDIAC20 & 7 & 41 \\
\hline INDIAC21 & 6 & 41 \\
\hline INDIAC22 & 6 & 41 \\
\hline INDIAC23 & 6 & 41 \\
\hline INDIAC24 & 6 & 36 \\
\hline INDIAC25 & 36 \\
\hline INDIAC26 & 36 \\
\hline INDIAC27 & & 36 \\
\hline NDIAC28 & 6 & \\
\hline
\end{tabular}




\section{INDIAN VARIANTS with embedded mutations sorted by percent frequencies}

The number of 17711 Fibonacci metastructures INCREASES with cumulated MUTATIONS

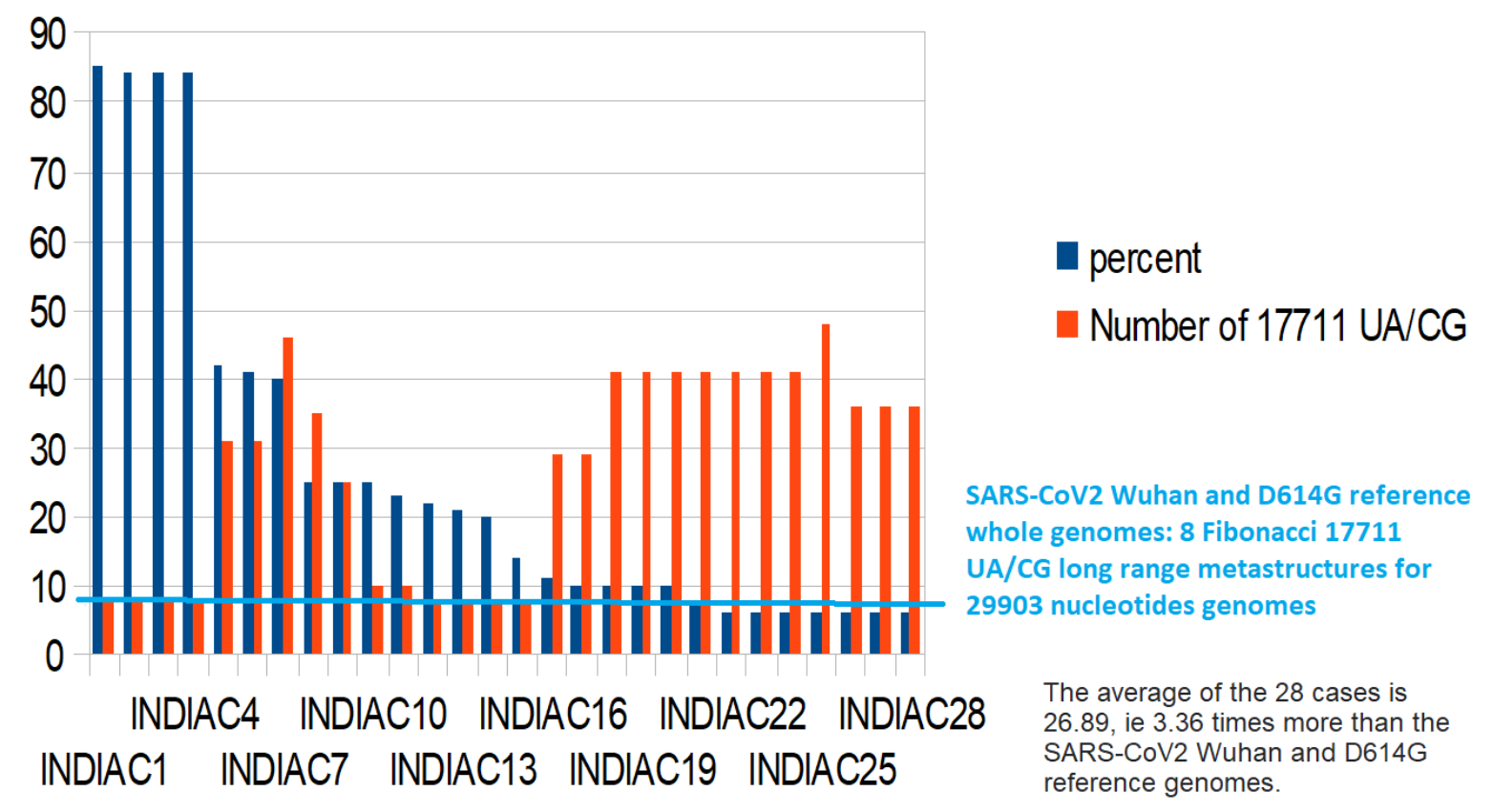

Figure 9 - Increase of 17711 UA/CG metastructures with whole INDIAN variant genomes with cumulated mutations vs percent frequencies (vs SARS-CoV2 Wuhan).

From this analysis, we can draw 3 conclusions:

a / this is a simulation of genomes made from SARS-CoV2 and the most frequently encountered mutations in India. So, if it is certain that the first genomes exist in some patients, some others, towards the end of the list of 28 genomes, may not exist but could potentially emerge.

$\mathrm{b} /$ it is noted that none of the 28 cases found UA / CG metastructures of 177122 bases in quantity LESS than 8, a value which characterizes SARS-CoV2 Wuhan.

So, if there was no correlation between these Fibonacci metastructures and the evolution of variants, we should find cases less than 8 .

c / out of the 28 cases of genomes studied, 20 of them saw an increase in the number of metastructures of 17,712 bases, or more than $2 / 3$ of the genomes studied. The average of the 28 cases is 26.89 , ie 3.36 times more than the SARS-CoV2 Wuhan and D614G reference genomes.

\section{2 - INDIAN VARIANTS SIMULATIONS with mutations on B.1.617 variant :}

The strain of the variant B.1.617 has grown exponentially in India since the beginning of 2021. We are going to redo the 28 previous analyzes no longer from the SARS-CoV2 Wuhan genome but by inserting the SINDIAFULL spike already analyzed in (Perez, 2021). 
This therefore amounts to applying the successive mutations to a type B 1.617 genome, at least at the level of its Spike sequence.

Indeed,

\section{B.1.617 lineage}

This strain, also known as the "double mutant virus", has spread rapidly through India.

The strain has been dubbed the "double mutant virus" due to two of the concerning mutations it carries.

These two key mutations are:

- E484Q

- L452R

Further studies on the strain are needed to determine its transmissibility, although it is suspected to do so due to its spike protein mutations which are thought to increase immune evasion and receptor binding. Whether vaccine efficacy is affected also needs further research.

SINDIAFULL is the Spike B.1.617 from (Perez-2021).

Recall Spike location

21563..25384

$$
\text { /gene="S" }
$$

$1 / 2$ V,,VSARSCOV2REF[21562+1/4(25384-21562)]

3822

$\mathrm{V}[1 / 49]$

ATGTTTGTT

$1 / 2$ SINDIAFULL

3822

SINDIAFULL[1/49]

ATGTTTGTT

VB1617,,VSARSCOV2REF

$1 / 2$ VB1617[21562+1/4(25384-21562)],,SINDIAFULL

3822

+/VB1617ᄀVSARSCOV2REF

8

(VB1617 $\neg$ VSARSCOV2REF)/VSARSCOV2REF

GAGGTGAA

(VB1617 $\neg$ VSARSCOV2REF)/VB1617

TCTTGATG

R,,GFIBOZOOMS VB1617

VINDIAC1,,VB1617

VINDIAC1[23403],'G'

VINDIAC2,,VINDIAC1

VINDIAC2[3037],,'T'

VINDIAC3,,VINDIAC2

VINDIAC3[241],,'T'

VINDIAC4,,VINDIAC3

VINDIAC4[14408],,'T'

VINDIAC5,,VINDIAC4

VINDIAC5[28881],'A' 
VINDIAC6,,VINDIAC5

VINDIAC6[28883],'C'

VINDIAC7,,VINDIAC6

VINDIAC7[28882],'A'

VINDIAC8,,VINDIAC7

VINDIAC8[25563],,'T'

VINDIAC9,,VINDIAC8

VINDIAC9[18877],,'T'

VINDIAC10,,VINDIAC9

VINDIAC10[26735],'T'

VINDIAC11,,VINDIAC10

VINDIAC11[28854],,'T'

VINDIAC12,,VINDIAC11

VINDIAC12[22444],,'T'

VINDIAC13,,VINDIAC12

VINDIAC13[313],'T'

VINDIAC14,,VINDIAC13

VINDIAC14[5700],,'A'

VINDIAC15,,VINDIAC14

VINDIAC15[11083],,'T'

VINDIAC16,,VINDIAC15

VINDIAC16[13730],,'T'

VINDIAC17,,VINDIAC16

VINDIAC 17[28311],,'T'

VINDIAC18,,VINDIAC17

VINDIAC18[23929],,'T'

VINDIAC19,,VINDIAC18

VINDIAC19[6312],'A'

VINDIAC20,,VINDIAC19

VINDIAC20[8917],,'T'

Table 5 - Summary on the 28 most frequent India country mutations applied to B.1.617 genome.

\begin{tabular}{|l|l|l|l|l|l|l|l|}
\hline position & $\begin{array}{l}\text { Genome } \\
\text { location }\end{array}$ & ref & alt & gene & $\begin{array}{l}\text { Amino Acids } \\
\text { mutations }\end{array}$ & Percent & $\begin{array}{l}\text { Number of } \\
\text { 17711 UA/CG } \\
\text { metastructures }\end{array}$ \\
\hline $\begin{array}{l}\text { SARS-CoV2 } \\
\text { Wuhan }\end{array}$ & & & & & 8 \\
\hline B1617 & & & & & & & 31 \\
\hline INDIAC1 & 23403 & A & G & "S:614" & "D614G" & 85 & 31 \\
\hline INDIAC2 & 3037 & C & T & "ORF1a:924" & "F924F" & 84 & 31 \\
\hline INDIAC3 & 241 & C & T & "5'UTR" & "NA" & 84 & 31 \\
\hline INDIAC4 & 14408 & C & T & "ORF1b:314" & "P314L" & 84 & 46 \\
\hline INDIAC5 & 28881 & G & A & "N:203" & "R203K" & 42 & 35 \\
\hline INDIAC6 & 28883 & G & C & "N:204" & "G204R" & 41 & 35 \\
\hline INDIAC7 & 28882 & G & A & "N:203" & "R203K" & 40 & 25 \\
\hline INDIAC8 & 25563 & G & T & "ORF3a:57" & "Q57H" & 25 & 10 \\
\hline INDIAC9 & 18877 & C & T & "ORF1b:1804" & "L1804L" & 25 & 10 \\
\hline INDIAC10 & 26735 & C & T & "M:71" & "Y71Y" & 25 & 8 \\
\hline INDIAC11 & 28854 & C & T & "N:194" & "S194L" & 23 & 29 \\
\hline
\end{tabular}




\begin{tabular}{|l|l|l|l|l|l|l|l|}
\hline INDIAC12 & 22444 & $\mathrm{C}$ & $\mathrm{T}$ & "S:294" & "D294D" & 22 & 29 \\
\hline INDIAC13 & 313 & $\mathrm{C}$ & $\mathrm{T}$ & "ORF1a:16" & "L16L" & 21 & 29 \\
\hline INDIAC14 & 5700 & $\mathrm{C}$ & $\mathrm{A}$ & "ORF1a:1812" & "A1812D" & 20 & 29 \\
\hline INDIAC15 & 11083 & $\mathrm{G}$ & $\mathrm{T}$ & "ORF1a:3606" & "L3606F" & 14 & 29 \\
\hline INDIAC16 & 13730 & $\mathrm{C}$ & $\mathrm{T}$ & "ORF1b:88" & "A88V" & 11 & 41 \\
\hline INDIAC17 & 28311 & $\mathrm{C}$ & $\mathrm{T}$ & "N:13" & "P13L" & 10 & 48 \\
\hline INDIAC18 & 23929 & $\mathrm{C}$ & $\mathrm{T}$ & "S:789" & "Y789Y" & 10 & 36 \\
\hline INDIAC19 & 6312 & $\mathrm{C}$ & $\mathrm{A}$ & "ORF1a:2016" & "T2016K" & 10 & 36 \\
\hline INDIAC20 & 8917 & $\mathrm{C}$ & $\mathrm{T}$ & "ORF1a:2884" & "F2884F" & 10 & 36 \\
\hline INDIAC21 & 1947 & $\mathrm{~T}$ & $\mathrm{C}$ & "ORF1a:561" & "V561A" & 7 & 36 \\
\hline INDIAC22 & 9389 & $\mathrm{G}$ & $\mathrm{A}$ & "ORF1a:3042" & "D3042N" & 6 & 36 \\
\hline INDIAC23 & 6573 & $\mathrm{C}$ & $\mathrm{T}$ & "ORF1a:2103" & "S2103F" & 6 & 36 \\
\hline INDIAC24 & 4354 & $\mathrm{G}$ & $\mathrm{A}$ & "ORF1a:1363" & "E1363E" & 6 & 36 \\
\hline INDIAC25 & 25528 & $\mathrm{C}$ & $\mathrm{T}$ & "ORF3a:46" & "L46F" & 6 & 34 \\
\hline INDIAC26 & 15324 & $\mathrm{C}$ & $\mathrm{T}$ & "ORF1b:619" & "N619N" & 6 & 62 \\
\hline INDIAC27 & 3267 & $\mathrm{C}$ & $\mathrm{T}$ & "ORF1a:1001" & "T1001I" & 6 & 62 \\
\hline INDIAC28 & 3634 & $\mathrm{C}$ & $\mathrm{T}$ & "ORF1a:1123" & "N1123N" & 6 & 62 \\
\hline Average & & & & & & $\mathbf{2 6 . 2 5}$ & $\mathbf{3 4 . 5 7}$ \\
\hline & & & & & $\mathbf{6}$ & \\
\hline
\end{tabular}

Table 6 - Recall summary main results from Table5. 


\begin{tabular}{l|r|r|} 
Genome & percent & Number of 1 \\
\hline SARS-CoV2 Wuhan & 8 \\
\hline B.1.617 & & 31 \\
\hline INDIAC1 & 85 & 31 \\
\hline INDIAC2 & 84 & 31 \\
\hline INDIAC3 & 84 & 31 \\
\hline INDIAC4 & 84 & 46 \\
\hline INDIAC5 & 42 & 35 \\
\hline INDIAC6 & 41 & 35 \\
\hline INDIAC7 & 40 & 25 \\
\hline INDIAC8 & 25 & 10 \\
\hline INDIAC9 & 25 & 10 \\
\hline INDIAC10 & 25 & 8 \\
\hline INDIAC11 & 23 & 29 \\
\hline INDIAC12 & 22 & 29 \\
\hline INDIAC13 & 21 & 29 \\
\hline INDIAC14 & 20 & 29 \\
\hline INDIAC15 & 14 & 29 \\
\hline INDIAC16 & 11 & 41 \\
\hline INDIAC17 & 10 & 48 \\
\hline INDIAC18 & 10 & 36 \\
\hline INDIAC19 & 10 & 36 \\
\hline INDIAC20 & 10 & 36 \\
\hline INDIAC21 & 7 & 36 \\
\hline INDIAC22 & 6 & 36 \\
\hline INDIAC23 & 6 & 36 \\
\hline INDIAC24 & 6 & 36 \\
\hline INDIAC25 & 6 & 34 \\
\hline INDIAC26 & 6 & 62 \\
\hline INDIAC27 & 6 & 62 \\
\hline NDIAC28 & 6 & 62 \\
\hline
\end{tabular}




\section{INDIAN B.1617 Variant combined with most frequent INDIA MUTATIONS}

\section{The number of 17711 UA/CG Fibonacci metastructures is 4.3 Times more ref Wuhan $\S D 654$}

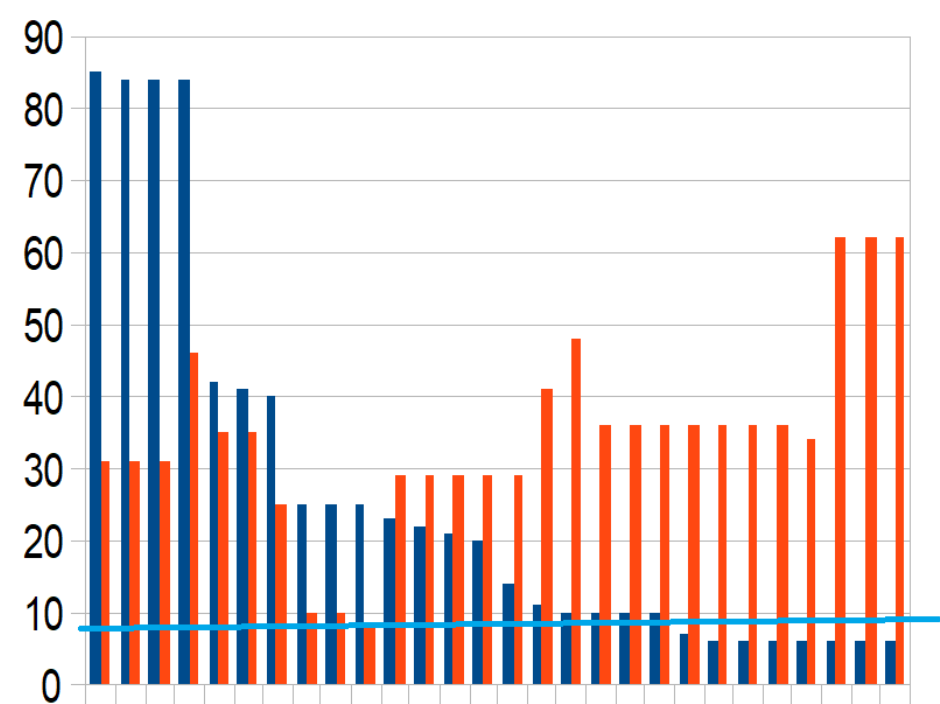

We note that out of the 28 genomes studied only one of them possesses the 8 characteristic metastructures of SARS-COV2 Wuhan.

The average of the other 27 is 34.57 , ie 4.32 times more and some cases are 8 times more INDIA26-28 : 62 Fibonacci 17711 UA/CG).

\section{percent}

Number of 17711 UA/CG

\section{SARS-CoV2 Wuhan and D614G reference} whole genomes: 8 Fibonacci 17711 UA/CG long range metastructures for 29903 nucleotides genomes

Figure 10 - Increase of $17711 \mathrm{UA} / \mathrm{CG}$ metastructures with whole INDIAN variant genomes with cumulated mutations vs percent frequencies (vs. B.1.617 variant).

The most remarkable result is the fact that the very simple combination of the 4 most frequent mutations ( $85 \%$ of cases) and the variant B.1.617 is sufficient to multiply by 4 to 6 (31 to 46 against 8 for SARS-CoV2 Wuhan (the number of Fibonacci metastructures of $17,712 \mathrm{AU} / \mathrm{CG}$ bases. We also note that out of the 28 genomes studied, only one of them possesses the 8 characteristic metastructures of SARS-COV2 Wuhan. The average of the other 27 is 34.57 , ie 4.32 times more and some cases are 8 times more INDIA26-28:62). 


\section{Whole GENOME SARS-COV2 WUHAN Collected Jan. 2020}

FIBONACCI UCAG Metastructures

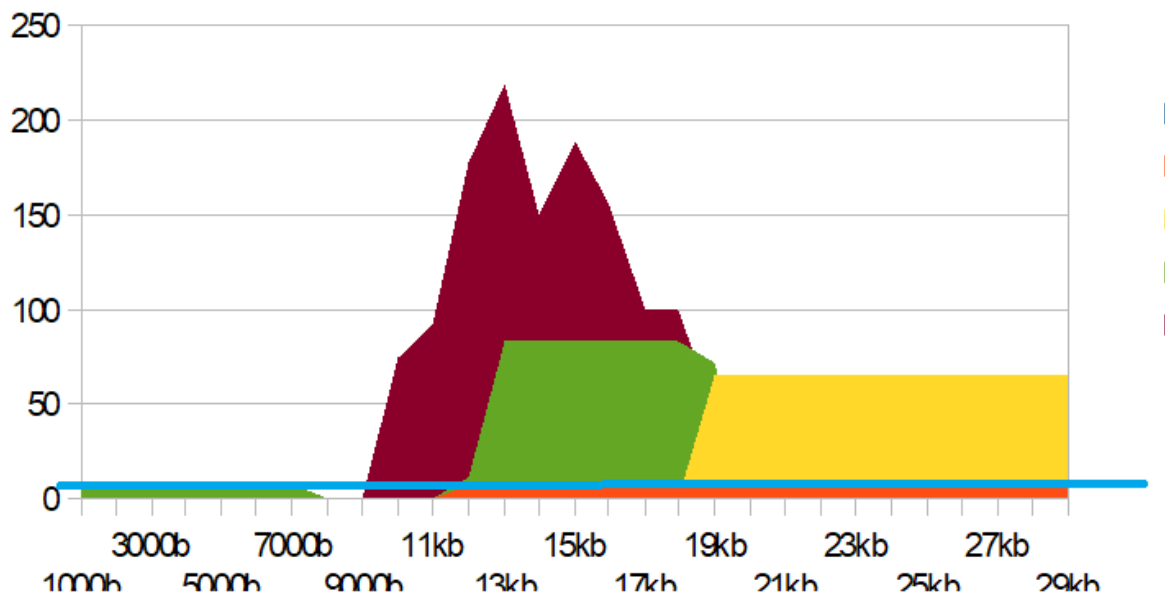

$10946 \mathrm{~b}$

$6765 b$

प4181b

SARS-CoV2 Wuhan reference genome (8 Fibonacci 17711 $\mathrm{UA} / \mathrm{CG})$

\section{INDI28: Variant B.1.617 with the 28 frequently Inda mutations}

\section{E2longrange Fbonacci 17711 UACG metastuctures}

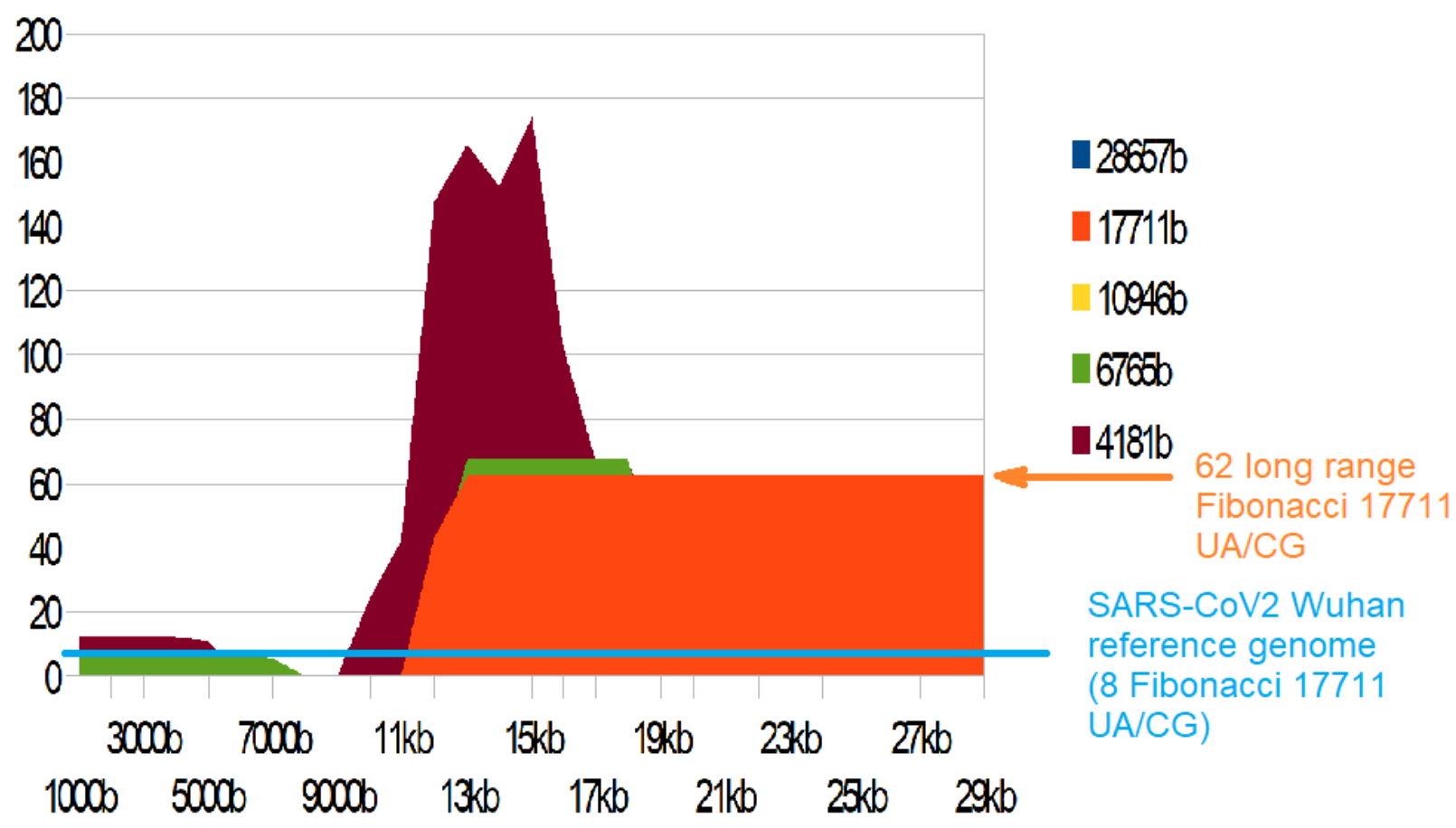

Figure 11 - Comparing long range 17711 UA/CG Fibonacci metastructures between SARS-CoV2 Wuhan and India variant B.1.617 with the 28 most frequent India country mutations. 


\section{3 - simulations of possible future mutations of the variant B.1.617.}

In (Pragya Yadav et al, 2021), the authors provide a list of the 33 main mutations characterizing the genomes of the Indian variant B 1.617.

On the other hand, we have just studied the impact of the 28 most frequent mutations in India, those which represent more than 5\% of contaminations).

It is clear that these 2 sets of mutations partially overlap.

However it would be interesting to simulate the effect of some of the 28 mutations when they are absent in B.1.617. Indeed, their high frequency makes it possible to suggest their possible future addition to B.1.617.

This is what we will simulate in this last paragraph.

Table 7 - The 33 main muatations from India variant B.1.617 from (Pragya Yadav et al., 2021). From Figure 1:nCharacteristics and neutralization of VUI B.1.617 variant: A)nThe common nucleotide changes observed in majority of the isolates and clinical sequences. We identify 22 other frequent mutations in India (frequency greater than $5 \%$ of contaminations) but absent in the Indian variant B.1.617.

\begin{tabular}{|c|c|c|c|c|}
\hline $\begin{array}{l}\text { Genome } \\
\text { location }\end{array}$ & $\begin{array}{l}\text { Reference } \\
\text { SARS-CoV2 }\end{array}$ & $\begin{array}{l}\text { Mutation } \\
\text { B.1.617 }\end{array}$ & Percent & $\begin{array}{l}17711 \text { UA/CG } \\
\text { Fibonacci } \\
\text { metastructures }\end{array}$ \\
\hline B1.1617 & \multicolumn{2}{|c|}{$\begin{array}{c}\text { All } 32 \text { following } \\
\text { mutations }\end{array}$} & & 53 \\
\hline $210 \mathrm{GT}$ & G & $T$ & & \\
\hline $3457 \mathrm{CT}$ & C & $T$ & & \\
\hline $11201 \mathrm{CT}$ & C & $T$ & & \\
\hline $16134 \mathrm{CT}$ & C & $T$ & & \\
\hline $20396 \mathrm{CT}$ & C & $T$ & & \\
\hline 21895 GA & G & A & & \\
\hline 22917 AG & A & G & & \\
\hline $23604 \mathrm{CT}$ & C & $T$ & & \\
\hline $26767 \mathrm{GA}$ & G & A & & \\
\hline $27520 \mathrm{CT}$ & C & $T$ & & \\
\hline 29402 GT & G & $T$ & & \\
\hline $241 \mathrm{GT} \quad$ INDIAC3 & G & $T$ & & \\
\hline $4965 \mathrm{AG}$ & A & G & & \\
\hline 14408 TG INDIAC4 & $T$ & G & & \\
\hline $16852 \mathrm{CT}$ & C & $T$ & & \\
\hline $20401 \mathrm{TC}$ & $T$ & C & & \\
\hline 21987 GA & G & A & & \\
\hline $23012 \mathrm{GA}$ & G & A & & \\
\hline 24775 TG & $T$ & G & & \\
\hline $27382 \mathrm{GC}$ & G & C & & \\
\hline
\end{tabular}




\begin{tabular}{|c|c|c|c|c|}
\hline 27638 AG & A & G & & \\
\hline 29742 CG & C & G & & \\
\hline 3037 AT INDIAC2 & A & $T$ & & \\
\hline 8491 CT & C & $\mathrm{T}$ & & \\
\hline 14772 TG & $\mathrm{T}$ & G & & \\
\hline 17523 GC & G & C & & \\
\hline 21846 TC & $\mathrm{T}$ & C & & \\
\hline 22022 AT & A & $\mathrm{T}$ & & \\
\hline 23403 TC INDIAC1 & $\mathrm{T}$ & C & & \\
\hline $25469 \mathrm{GT}$ & G & $\mathrm{T}$ & & \\
\hline 27385 GT & G & $\mathrm{T}$ & & \\
\hline 28881 GT INDIAC5 & G & $T$ & & \\
\hline \multicolumn{5}{|c|}{ Other mutations common in India but absent in the Indian variant B.1.617 } \\
\hline 28883 SINDIAC6 & G & $\mathrm{C}$ & 41 & 53 \\
\hline 28882 SINDIAC7 & G & A & 40 & 32 \\
\hline 25563 SINDIAC8 & G & $\mathrm{T}$ & 25 & 21 \\
\hline 26735 SINDIAC10 & $\mathrm{C}$ & $\mathrm{T}$ & 25 & 14 \\
\hline 28854 SINDIAC11 & C & $\mathrm{T}$ & 23 & 8 \\
\hline 22444 SINDIAC12 & $\mathrm{C}$ & $\mathrm{T}$ & 22 & 31 \\
\hline 313 SINSDIAC13 & $\mathrm{C}$ & $\mathrm{T}$ & 21 & 31 \\
\hline 5700 SINDIAC14 & $\mathrm{C}$ & A & 20 & 31 \\
\hline 11083 SINDIAC15 & G & $\mathrm{T}$ & 14 & 31 \\
\hline 13730 SINDIAC16 & $\mathrm{C}$ & $\mathrm{T}$ & 11 & 28 \\
\hline 28311 SINDIAC17 & $\mathrm{C}$ & $\mathrm{T}$ & 10 & 40 \\
\hline 23929 SINDIAC18 & $\mathrm{C}$ & $\mathrm{T}$ & 10 & 48 \\
\hline 6312 SINDIAC19 & $\mathrm{C}$ & A & 10 & 48 \\
\hline 8917 SINDIAC20 & $\mathrm{C}$ & $\mathrm{T}$ & 10 & 48 \\
\hline 1947 SINDIAC21 & $\mathrm{T}$ & C & 7 & 48 \\
\hline 9389 SINDIAC22 & G & A & 6 & 48 \\
\hline 6573 SINDIAC23 & $\mathrm{C}$ & $\mathrm{T}$ & 6 & 48 \\
\hline 4354 SINDIAC24 & G & A & 6 & 48 \\
\hline 25528 SINDIAC25 & $\mathrm{C}$ & $\mathrm{T}$ & 6 & 38 \\
\hline 15324 SINDIAC26 & $\mathrm{C}$ & $\mathrm{T}$ & 6 & 45 \\
\hline 3267 SINDIAC27 & $\mathrm{C}$ & $\mathrm{T}$ & 6 & 45 \\
\hline 3634 SINDIAC28 & $\mathrm{C}$ & $T$ & 6 & 45 \\
\hline Average & & & $15.05 \%$ & 37.68 \\
\hline
\end{tabular}

Nota:We rename these successive mutants SINDIA6 for B.1.617 consensus + INDIA6 etc ... 
Analysing the 32 mutations concensus India variant B.617 :

We run here the 32 mutations applied to SARS-CoV2 reference Wuhan genome :

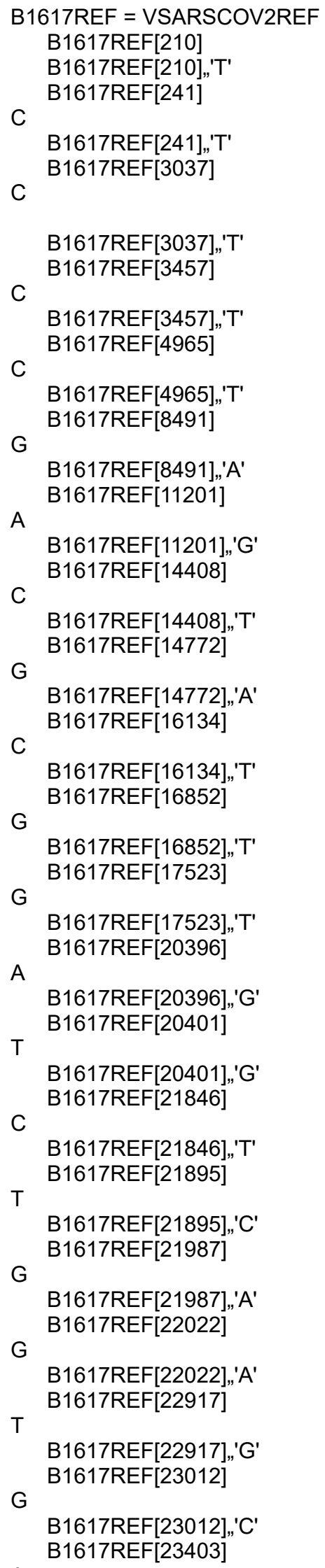


B1617REF[23403],'G'

B1617REF[23604]

C

B1617REF[23604],',G'

B1617REF[24775]

A

B1617REF[24775],'T'

C

B1617REF[25469]

B1617REF[25469],"'T'

B1617REF[26767]

$\mathrm{T}$

B1617REF[26767],,'G'

B1617REF[27382]

G

B1617REF[27382],"C'

B1617REF[27385]

$T$

B1617REF[27385],"C'

B1617REF[27520]

A

B1617REF[27520],' 'T'

B1617REF[27638]

T

B1617REF[27638],"C'

$G$

B1617REF[28881]

B1617REF[28881],,'T'

$G$

B1617REF[28881],,
B1617REF[29402]

B1617REF[29402],"'T'

B1617REF[29742]

G

B1617REF[29742],,'T' 
FIBONACCI UCAG Metastructures

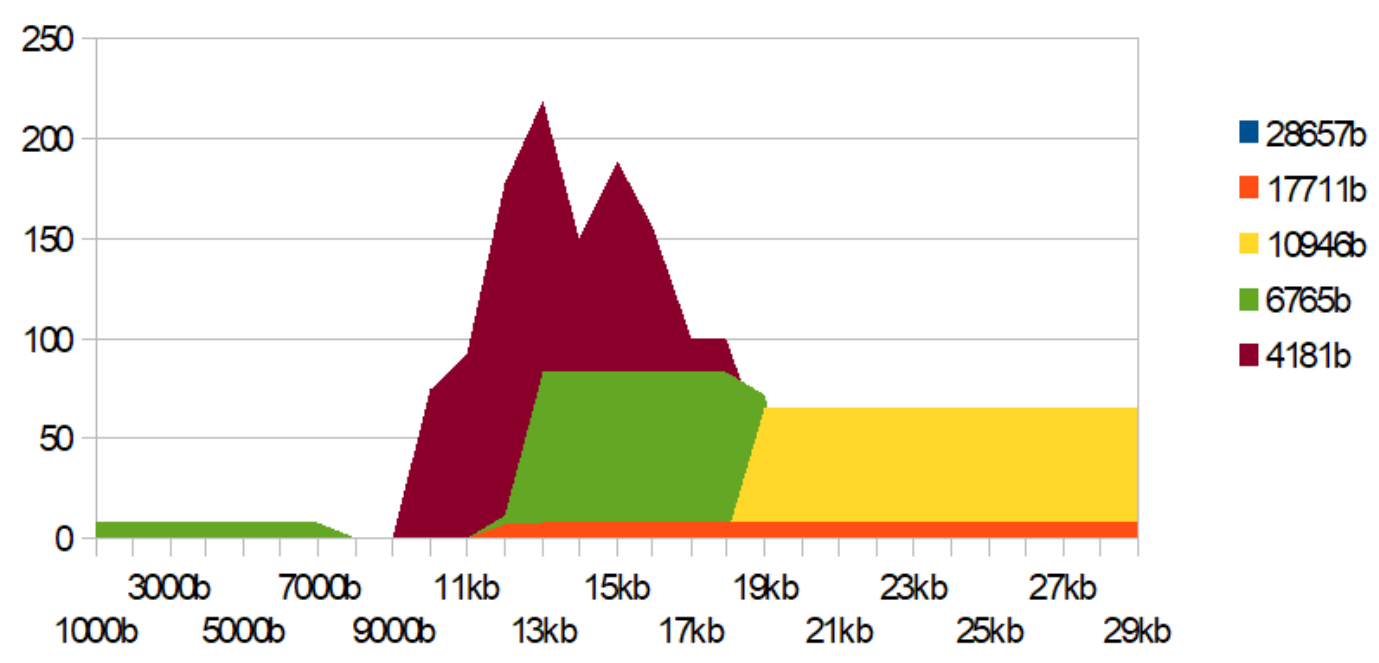

\section{INDIA Variant B.1.617 reference consensus}

\section{UACG Fibonacci metastructures overlaping whde genome}

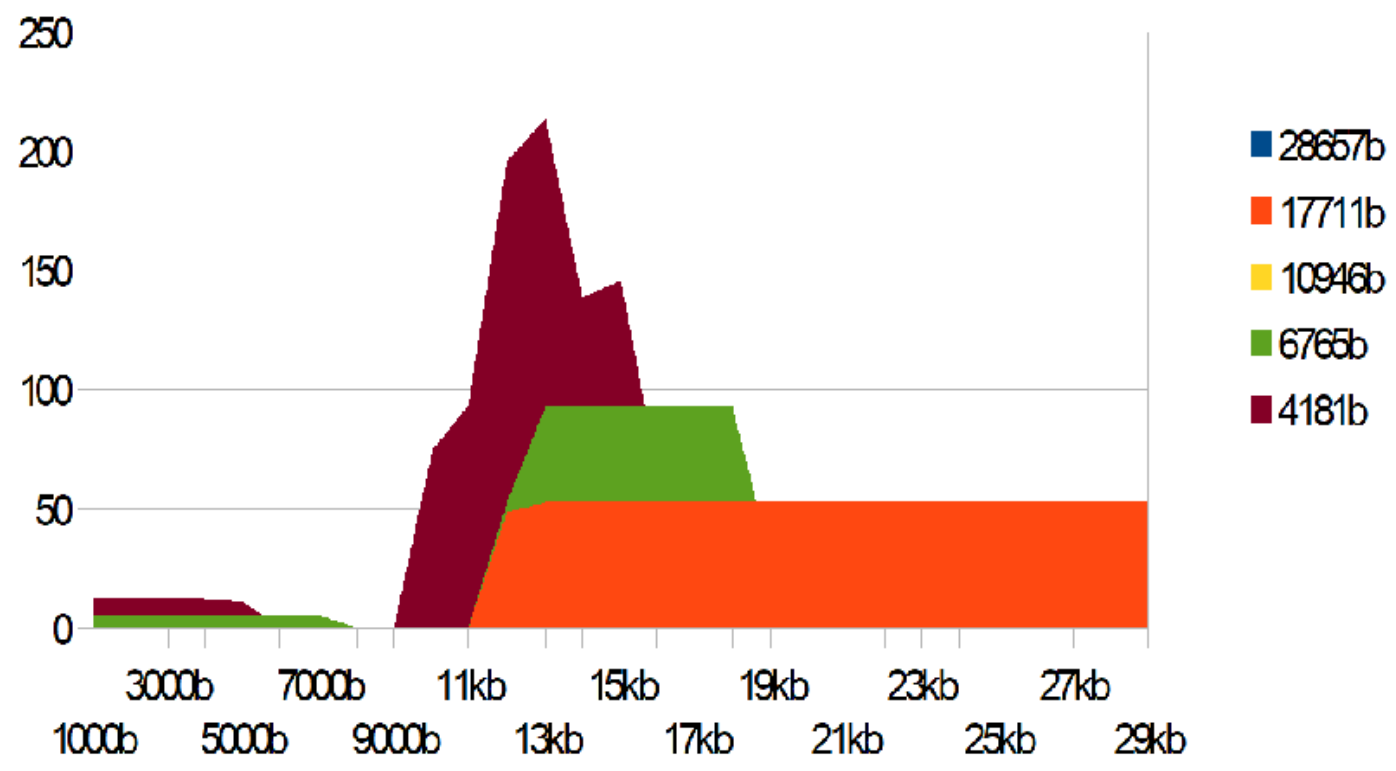

Comparing 17711 UA/CG Fibonacci metastructures between SARS-CoV2 Wuhan and INDIA VARIANT B.1.617 reference concensus whole genomes

Figure 12 - Comparing long range 17711 UA/CG Fibonacci metastructures between SARS-CoV2 Wuhan and India variant B.1.617 Reference Concensus ( Pragya Yadav et al, 2021 ) including 32 mutations.

Now we will apply to this strain B.1.617 consensus the progressive accumulation of the 22 other frequent mutations in India (frequency greater than $5 \%$ of contaminations) but absent in the Indian variant B.1.617. 
For this purpose, as we did in the previous $\S$, we will apply to B.1.617 consensus each of the 22 mutations, accumulating them one by one and respecting the order of their frequency of contamination in India (here in the order INDIAC6, then INDIAC6 + INDIAC7, then INDIAC6 + INDIAC7 + INDIAC8 ... as these muattions appear in Table 7.

Nota:We rename these successive mutants SINDIA6 for B.1.617 consensus + INDIA6 etc...

G

SINDIAC6,B1617REF

SINDIAC6[28883]

SINDIAC6[28883],'C'

SINDIAC7,SINDIAC6

G

SINDIAC7[28882]

SINDIAC8,SINDIAC7

SINDIAC7[28882],'A'

G SINDIAC8[25563]

SINDIAC8[25563],'T'

SINDIAC10, SINDIAC8 SINDIAC10[26735]

C SINDIAC10[26735],'T'

SINDIAC11,SINDIAC10 SINDIAC11[28854] SINDIAC11[28854],'T'

SINDIAC12,,SINDIAC11

C SINDIAC12[22444]

SINDIAC12[22444],,'T'

SINDIAC13,,SINDIAC12

C SINDIAC13[313]

SINDIAC13[313],'T'

SINDIAC14,,SINDIAC13

C SINDIAC14[5700]

SINDIAC14[5700],'A'

SINDIAC15,,SINDIAC14

G SINDIAC15[11083]

SINDIAC15[11083],'T'

SINDIAC16,,SINDIAC15

C SINDIAC16[13730]

SINDIAC16[13730],'T'

SINDIAC17,SINDIAC16

C SINDIAC17[23929]

SINDIAC17[28311],'T'

SINDIAC18,SINDIAC17

C

SINDIAC18[23929]

SINDIAC18[23929],'T'

SINDIAC19,,SINDIAC18

C

SINDIAC19[6312]

SINDIAC19[6312],'A'

SINDIAC20,,SINDIAC19 
C SINDIAC20[8917] SINDIAC20[8917],''T'

SINDIAC21,,SINDIAC20

SINDIAC21[1947]

$T$

SINDIAC21[1947],"'C'

SINDIAC22,,SINDIAC21

G SINDIAC22[9389]

SINDIAC22[9389],,'A'

SINDIAC23,,SINDIAC22

C SINDIAC23[6573]

SINDIAC23[6573],''T'

SINDIAC24,,SINDIAC23

G SINDIAC24[4354]

SINDIAC24[4354],'A'

SINDIAC25,SINDIAC24

C SINDIAC25[25528]

SINDIAC25[25528],'T'

SINDIAC26,,SINDIAC25

C SINDIAC26[15324]

SINDIAC26[15324],,'T'

SINDIAC27,,SINDIAC26

C SINDIAC27[3267] SINDIAC27[3267],''T'

SINDIAC28,SINDIAC27

C SINDIAC28[3634] SINDIAC28[3634],'T'

Table 8 - Evolution of 17711 UA/CG metastructures with whole INDIAN variant genomes with cumulated mutations vs percent frequencies (vs. B.1.617 REF variant). 


\begin{tabular}{|c|c|c|}
\hline Genome & percent & Number of 1 \\
\hline SARS-CoV2 & 2 Wuhan & 0 \\
\hline B.1.617 REF & & 53 \\
\hline 28883 SIN & 41 & 53 \\
\hline 28882 SIN & 40 & 32 \\
\hline 25563 SIN & 25 & 21 \\
\hline 26735 SIN & 25 & 14 \\
\hline 28854 SIN & 23 & 8 \\
\hline 22444 SIN & 22 & 31 \\
\hline 313 SINSD & 21 & 31 \\
\hline 5700 SIND & 20 & 31 \\
\hline 11083 SIN & 14 & 31 \\
\hline 13730 SIN & 11 & 28 \\
\hline 28311 SIN & 10 & 40 \\
\hline 23929 SIN & 10 & 48 \\
\hline 6312 SIND & 10 & 48 \\
\hline 8917 SIND & 10 & 48 \\
\hline 1947 SIND & 7 & 48 \\
\hline 9389 SIND & 6 & 48 \\
\hline 6573 SIND & 6 & 48 \\
\hline 4354 SIND & 6 & 48 \\
\hline 25528 SIN & 6 & 38 \\
\hline 15324 SIN & 6 & 45 \\
\hline 3267 SIND & 6 & 45 \\
\hline 3634 SIND & 6 & 45 \\
\hline
\end{tabular}

Here, unlike the 2 previous simulations where most of the mutations INCREASED the number of long AU / CG metastructures, here almost all of the mutations DECREASE the number of these long metastructures. It is true that the level of these metastructures of 17711 AU / CG bases is very IMPORTANT in the reference genome B.1.617 Ref.

The level of the B.1.617 consensus reference variant genome is however more than 6.6 times higher than that of the Wuhan SARS-CoV2 reference genome.

The average level of these 22 nested mutations applied to the variant genome consensus reference B.1.617 is however more than 4.7 times higher than that of the reference genome SARS-CoV2 Wuhan. 


\section{Evolution of the 22 India country frequent mutations (>5\%) altering B.1.617 REF}

\section{Long range 17711 UA/CG Fibonacci metastructures}

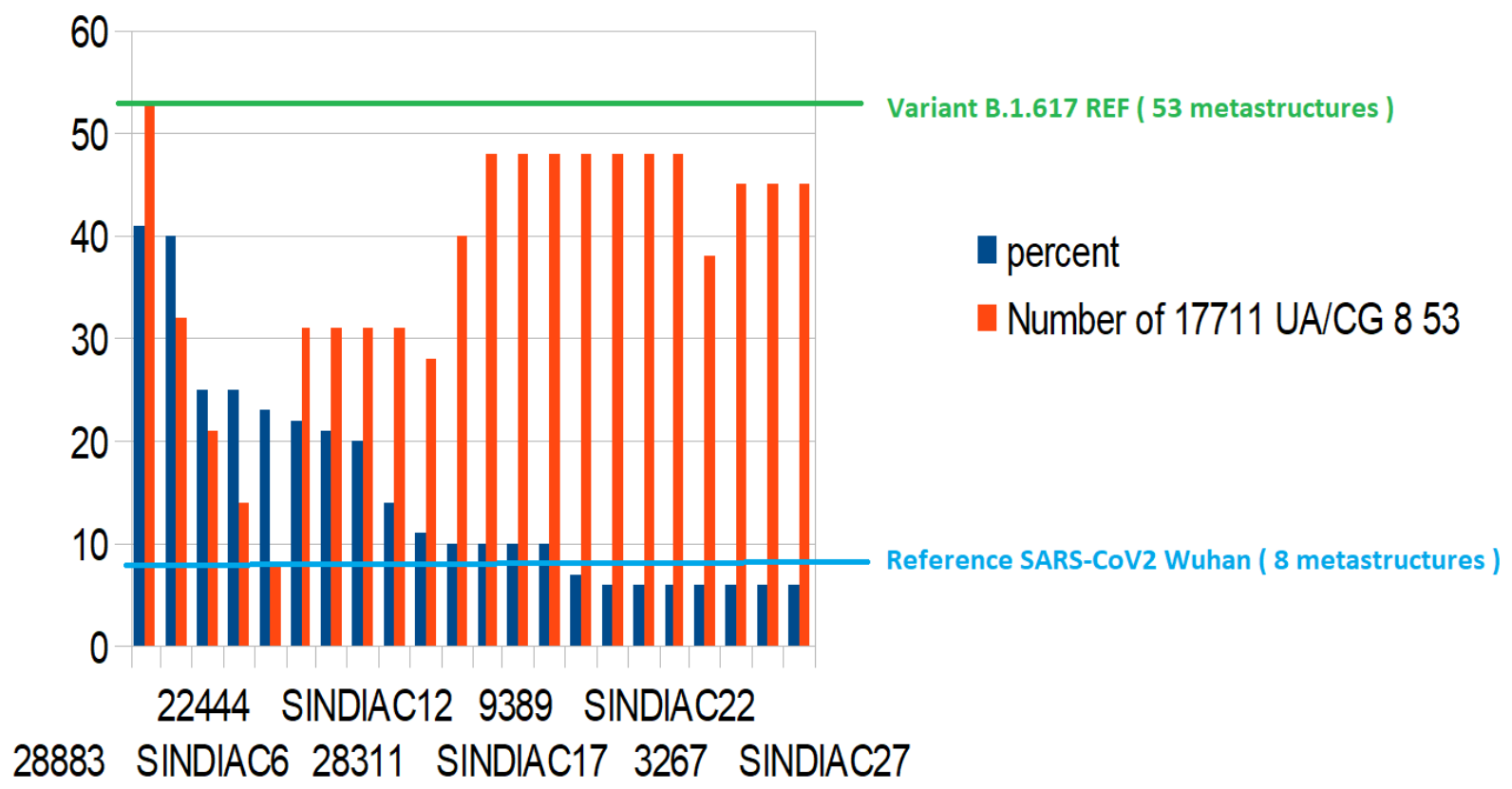

Figure 13 - Evolution of 17711 UA/CG metastructures with whole INDIAN variant genomes with cumulated mutations vs percent frequencies (vs. B.1.617 REF variant).

\section{CONCLUSIONS.}

In this study, we limit ourselves to the analysis of whole genomes, all coming from the mutations and variants of SARS-CoV2 sequenced in India in 2020 and 2021. We then demonstrate - both on actual genomes of patients and on variants combining the most frequent mutations to the SARS-CoV2 Wuhan genomes and then to the B.1.617 variant that the numerical Fibonacci AU / CG metastructures increase considerably in all cases analyzed in ratios of up to 8 times. We can affirm that this property contributes to a greater stability and lifespan of messenger RNAs, therefore, possibly also to a greater INFECTUOSITY of these variant genomes. 
Comparing India frequent mutations from SARS-CoV2 Wuhan and B.1.617 variant

\section{Number of long Fibonacci 17711 UA/CG metastructures}

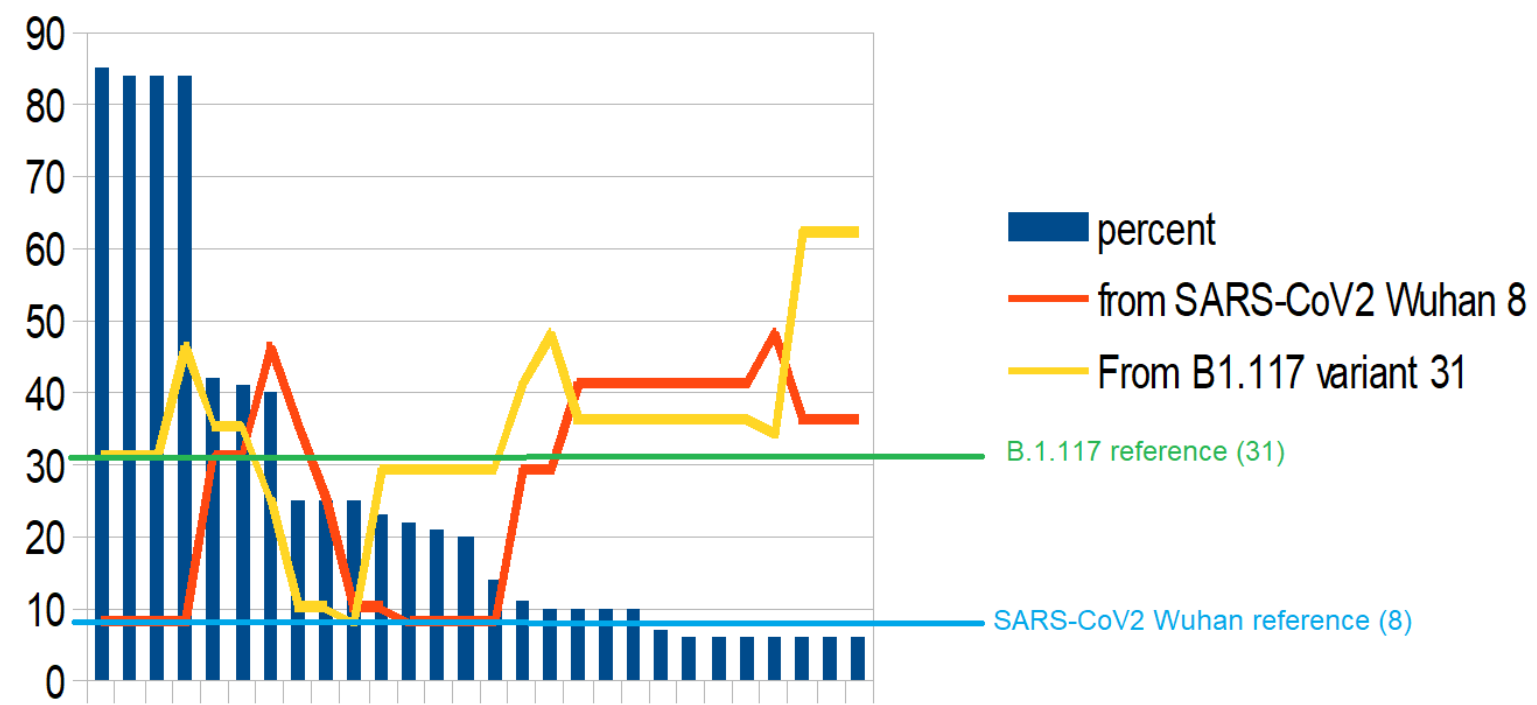

INDIAC4 INDIAC10 INDIAC16 INDIAC22 INDIAC28

INDIAC1 INDIAC7 INDIAC13 INDIAC19 INDIAC25

Figure 14 - summarizing both 28 SARS-CoV2 and 28 B.1.617 embedded mutations cases.

In this study, we looked for the presence and number of UA / CG Fibonacci metastructures. We are interested in the longest of 17,711 bases, for genomes of 29,000 bases. These genomes concerned, for some, real patients, and, for others, the 28 mutations and variants most frequent in India, those which represent more than $5 \%$ of the cases of infections of the country.

Out of a total of 87 genomes analyzed:

- None ("NONE") of them contained a number of metastructures LOWER than those of the reference SARS-CoV2 Wuhan genome.

- Eleven (11) among them contained the same number of metastructures as the reference genome.

- 76 of them contained a GREATER number of metastructures than the reference genome, ie $\mathbf{8 7 \%}$ of cases. The average increase in the number of metastructures for the 76 cases studied is 4.11 times the number of SARS-CoV2 UA/CG 17711 Fibonacci metastructures. $(4.11=32.86 / 8)$.

Note the impact of the new B.1.617 variant which, combined with the 4 most frequent mutations ( $85 \%$ of contaminations in the country), multiplies by 4 ("four") the number of metastructures of 17,711 bases compared to the reference genome SARS-CoV2 ( $8==>$ $31)$. It is therefore clear that the evolutionary pressure of mutations and variants operates on the mRNAs of viruses a sort of adaptation and even OPTIMIZATION of the AU / CG ratios of the entire genome. Only the virus "knows" this STRATEGY, and we think we have unveiled a corner of the veil here ...

When we run the most frequent mutations in India whole country, on the reference consensus B.1.617 India Variant, the level of the B.1.617 consensus reference 
variant genome is more than 6.6 times higher than that of the Wuhan SARS-CoV2 reference genome.

"We can add that the evolution of the virus towards the" Fibonacci "variants was favored by the anti-spike protein antibodies of the original SARS-CoV2 virus from Wuhan. Nature or God will not facilitate the reaction of vaccinators to end to this pandemic ". Luc Montagnier.

\section{V-ACKNOWLEDGEMENTS.}

Thanks for fructuous discussions about this article to Megawaty Tan (A private researcher based in South Sumatera, Indonesia), , Robert Freeman M D. (author of "Nature's secret nutrient, golden ratio biomimicry, for PEAK health, performance and longevity), Philippe Risby (initiator of "Learning to Survive") project in Portugal, Valère Lounnas, (Free lance researcher at CMBI European Molecular Biology Laboratory (EMBL) Heidelberg ), Dr Daniel Favre, independant researcher, Brent, Switzerland, Christian Marc, ( retired, MSEE-Dipl-Eng Physics, MBA (Beta Gamma Sigma, USA), Harvard HBS Alumn, General Director https://www.caravanedelapaix.com/), and Ethirajan Govindarajan (adjunct Professor, Department of Cybernetics, School of Computer Science, University of Petroleum and Energy Studies, Dehradun, Uttarakhand, India, Director, PRC Global Technologies Inc., Ontario, Canada, President, Pentagram Research Centre Pvt. Ltd., Hyderabad, India).

Particularly, this work is the result of multiple exchanges and advice, since the very beginning of the COVID-19 pandemic, for which I must thank Professor Luc Montagnier (Nobel prizewinner for his discovery of HIV, Fondation Luc Montagnier Quai GustaveAdor 621207 Geneva, Switzerland).

\section{REFERENCES.}

(Castro-Chavez, 2020), F. Castro-Chavez, (June 2020), Anticovidian v.2: COVID-19: Hypothesis of the Lab Origin versus a Zoonotic Event Which Can Also be of a Lab Origin, GJSFR, August 2020, https://zenodo.org/record/3988139\#.YGMMaq8zaM8

(Dae Eun Jeong et al, 2021), Dae Eun Jeong et al, Assemblies-of-putative-SARSCoV2-spike-encoding-mRNA-sequences-for-vaccines-BNT-162b2-and-mRNA1273, GitHub, March 2021, https://github.com/NAalytics/Assemblies-of-putative-SARSCoV2-spike-encoding-mRNA-sequences-for-vaccines-BNT-162b2-and-mRNA-1273

(Da Silva Filipe, 2020), da Silva Filipe, A., Shepherd, J.G., Williams, T. et al. Genomic epidemiology reveals multiple introductions of SARS-CoV-2 from mainland Europe into Scotland. Nat Microbiol 6, 112-122 (2021). https://doi.org/10.1038/s41564-020-00838-z

(Demongeot§Henrion-Caude, 2020), Demongeot J. § Henrion-Caude A. , Footprints of a Singular 22-Nucleotide RNA Ring at the Origin of Life, Biology 2020, 9(5),

88; https://doi.org/10.3390/biology9050088

( Gröhs Ferrareze P. A., et al, 2021), Patrícia Aline Gröhs Ferrareze, et al, E484K as an innovative phylogenetic event for viral evolution: Genomic analysis of the E484K spike mutation in SARSCoV-2 lineages from Brazil

bioRxiv 2021.01.27.426895; doi: https://doi.org/10.1101/2021.01.27.426895 
(Jackson et al, 2020), Jackson, N.A.C., Kester, K.E., Casimiro, D. et al. The promise of mRNA vaccines: a biotech and industrial perspective. npj Vaccines 5, 11 (2020). https://doi.org/10.1038/s41541-020-0159-8

(Kudla et al, 2016), Kudla, G., Lipinski, L., Caffin, F., Helwak, A. \& Zylicz, M. High guanine and cytosine content increases mRNA levels in mammalian cells. Plos Biol. 4, e180 (2016). High guanine and cytosine content increases mRNA levels in mammalian cells

(Kustin T. et al, 2021), Evidence for increased breakthrough rates of SARS-CoV-2 variants of concern in BNT162b2 mRNA vaccinated individuals, Talia Kustin et al, medRxiv Preprints, Doi: https://doi.org/10.1101/2021.04.06.21254882

(Mengwen et al, 2006), Mengwen Jia, Liaofu Luo, The relation between mRNA folding and protein structure, Biochemical and iophysical Research Communications, Volume 343, Issue 1,2006, Pages 177-182, ISSN 0006-291X, https://doi.org/10.1016/j.b.brc.2006.02.135. (https://www.sciencedirect.com/science/article/pii/s0006291X06004451)

(Montagnier L. § Kingsley Sanders F., 1963), Luc Montagnier and F. Kingsley Sanders « Replicative Form of Encephalomyocarditis virus RNA », Nature 199. 664-667. 1963

(Muttineri et al, 2021), Muttineni R, Kammili N, Bingi TC, Rao M. R, Putty K, Dholaniya PS, et al. (2021) Clinical and whole genome characterization of SARS-CoV-2 in India. PLoS ONE 16 (2): e0246173. doi: 10.1371 / journal.pone. 0246173

https://www.google.com/url?

sa=t\&source=web\&rct=j\&url=https://journals.plos.org/plospathogens/article/file\%3Fid \%3D10.1371/journal.pone.0246173\%26type \%3Dprintable\&ved=2ahUKEwj3zdnZnorwAhUQKBoKHUxnD EQFjABegQICBAC\&usg=A OvVaw1A79ux6UbetoPoRx_jT-Mk

(Naveca Felipe et al, 2021), Phylogenetic relationship of SARS-CoV-2 sequences from Amazonas with emerging Brazilian variants harboring mutations E484K and N501Y in the Spike protein, Virological.org, 2021, https://virological.org/t/phylogenetic-relationship-of-sars-cov-2sequences-from-amazonas-with-emerging-brazilian-variants-harboring-mutations-e484k-andn501y-in-the-spike-protein $/ \mathbf{5 8 5}$

(Perez, 1988), Perez J.C., De nouvelles voies vers I'Intelligence Artificielle, 1988, Ed. MASSON ELSEVIER, EAN 978-2225818158 ISBN 2225818150, https://livre.fnac.com/a223887/JeanClaude-Perez-De-Nouvelles-voies-vers-1-intelligence-artificielle

(Perez, 1991), J.C. Perez (1991), "Chaos DNA and Neuro-computers: A Golden Link", in Speculations in Science and Technologyvol. 14 no. 4, ISSN 0155-7785, January 1991 Speculations in Science and Cell Motility 14(4):155-7785

https://www.researchgate.net/publication/258439719 JC Perez_1991_Chaos_DNA and Neurocomputers_A_Golden_Link_in_Speculations_in_Science_and Technologyvol_14_no 4 ISSN_015 $\underline{5-7785}$

(Perez, 1997), Perez J.C, L'ADN décrypté, Ed. Marco Pietteur, ISBN : 2-87211-017-8 •EAN : 9782872110179, https://www.editionsmarcopietteur.com/resurgence/91-adn-decrypte9782872110179.html 
(Perez, 2009), Perez J.C, Codex biogenesis - Les 13 codes de l'ADN (French Edition) [Jean -Claude ... 2009);

Language: French; ISBN -10: 2874340448; ISBN -13: 978-2874340444

https://www.amazon.fr/Codex-Biogenesis-13-codes-1ADN/dp/2874340448

(Perez, 2015), Deciphering Hidden DNA Meta-Codes -The Great Unification \& Master Code of Biology, journal of Glycémies abd Lipidomics,

https://www.longdom.org/abstract/deciphering-hidden-dna-metacodes-the-great-unification-ampmaster-code-of-biology-11590.html , ISSN: 2153-0637, DOI: 10.4172/2153-0637.1000131

(Perez, 2017), J.C Perez, 2017, Sapiens Mitochondrial DNA Genome Circular Long Range Numerical Meta Structures are Highly Correlated with Cancers and Genetic Diseases mtDNA Mutations

January 2017 Journal of Cancer Science and Therapy 09(06) DOI: 10.4172/1948-5956.1000469

(Perez, 2017b), Jean Claude Perez, "The Master Code of Biology: Self-assembly of two identical Peptides beta A4 1-43 Amyloid In Alzheimerâ $€^{\mathrm{TM}}$ s Diseases," Biomedical Journal of Scientific \& Technical Research, Biomedical Research Network+, LLC, vol. 1(4), pages 1191-1195, September. Handle: RePEc:abf:journl:v:1:y:2017:i:4:p:1191-1195 DOI: 10.26717/BJSTR.2017.01.000394

(Perez, 2017c), Perez JC (2017) The "Master Code of DNA": Towards the Discovery of the SNPs Function (Single-Nucleotide Polymorphism). J Clin Epigenet. 3:26. doi: 10.21767/2472-1158.100060, https://clinical-epigenetics.imedpub.com/the-master-code-of-dna-towards-the-discovery-of-thesnps-function-singlenucleotide-polymorphism.pdf

(Perez, 2018), Perez, J.C. Six Fractal Codes of Biological Life:perspectives in Exobiology, Cancers Basic Research and Artificial Intelligence Biomimetism Decisions

Making. Preprints 2018, 2018090139 (doi: 10.20944/preprints201809.0139.v1). Perez, J.C.

Six Fractal Codes of Biological Life:perspectives in Exobiology, Cancers Basic Research and Artificial Intelligence Biomimetism Decisions Making. Preprints 2018, 2018090139 (doi:

10.20944/preprints201809.0139.v1).

https://www.preprints.org/manuscript/201809.0139/v1

(Perez, 2019) , Perez, J. Epigenetics Theoretical Limits of Synthetic Genomes: The Cases of Artificials Caulobacter (C. eth-2.0), Mycoplasma Mycoides (JCVI-Syn 1.0, JCVI-Syn 3.0 and JCVI_3A), E-coli and YEAST chr XII. Preprints 2019, 2019070120 (doi:

10.20944/preprints201907.0120.v1).

https://www.preprints.org/manuscript/201907.0120/v1

Perez J.C, (2020). “WUHAN COVID-19 SYNTHETIC ORIGINS AND

EVOLUTION.’International Journal of Research - Granthaalayah, 8(2), 285-324.

https://doi.org/10.5281/zenodo.3724003

(Perez§Montagnier, 2020a), Perez, j.c, \& Montagnier, L. (2020, April 25). COVID-19, SARS and Bats Coronaviruses Genomes unexpected Exogeneous RNA Sequences.

https://doi.org/10.31219/osf.io/d9e5g

(Perez\%Montagnier, 2020b), Jean claude Perez, \& Luc Montagnier. (2020). COVID-19, SARS AND BATS CORONAVIRUSES GENOMES PECULIAR HOMOLOGOUS RNA SEQUENCES. International Journal of Research -GRANTHAALAYAH ISSN (print): 2394-3629 July 2020, Vol 8(07), 217 - 263 DOI: 
Https://doi.org/10.29121/granthaalayah.v8.i7.2020.678, Vol 8(07), 217 - 263(Vol 8(07), 217 - 263), Vol 8(07), 217-Vol 8(07), 263. http://doi.org/10.5281/zenodo.3975578

(Perez, 2021) Perez, J. SARS-CoV2 Variants and Vaccines mRNA Spikes Fibonacci Numerical UA/CG Metastructures. Preprints 2021, 2021040034 (doi: 10.20944/preprints202104.0034.v5). Perez, J. SARS-CoV2 Variants and Vaccines mRNA Spikes Fibonacci Numerical UA/CG Metastructures. Preprints 2021, 2021040034 (doi: 10.20944/preprints202104.0034.v5).

https://www.preprints.org/manuscript/202104.0034/v5

(Pragya Yadav et al., 2021), Pragya Yadav, et al.,

Neutralization of variant under investigation B.1.617 with sera of BBV152 vaccinees

bioRxiv 2021.04.23.441101; doi: https://doi.org/10.1101/2021.04.23.441101

(Rapoport § Perez, 2018),

Rapoport D. § Perez J.C, Golden ratio and Klein bottle Logophysics: the Keys of the Codes of Life and Cognition.

Quantum Biosystems. 9(2) 8-76. ; Vol. 9 - n.2 - 2018

(Simmonds P, 2020), P. Simmonds, Rampant C->U hypermutation in the genomes of SARS-CoV-2 and other coronaviruses - causes and consequences for their short and long evolutionary trajectories. bioRxiv 2020.05.01.072330; doi: https://doi.org/10.1101/2020.05.01.072330

(Srivastava Surabhi et al, 2021), SARS-CoV-2 genomics: An Indian perspective on sequencing viral variants, J Biosci. 2021; 46(1): 22.

Published online 2021 Feb 20. doi: 10.1007/s12038-021-00145-7, https://www.ncbi.nlm.nih.gov/pmc/articles/PMC7895735/

( Wenjuan Zhang et al, 2021), Emergence of a novel SARS-CoV-2 strain in Southern California, USA, medRxiv 2021.01.18.21249786; doi: https://doi.org/10.1101/2021.01.18.21249786 